\title{
Threats to civilian aviation since 1975
}

\author{
Luke Kelly \\ University of Manchester \\ 5 February 2021
}

\section{Question}

- What are the major threats and risks to civilian aviation?

- What are the factors that increase / decrease these risks and threats?

\section{Contents}

1. Summary

2. Incidents

3. Factors shaping threats

4. References

Appendix: Trends in airline fatalities and hijackings, 1975-2017 (graphs)

The K4D helpdesk service provides brief summaries of current research, evidence, and lessons learned. Helpdesk reports are not rigorous or systematic reviews; they are intended to provide an introduction to the most important evidence related to a research question. They draw on a rapid desk-based review of published literature and consultation with subject specialists.

Helpdesk reports are commissioned by the UK Foreign, Commonwealth, and Development Office and other Government departments, but the views and opinions expressed do not necessarily reflect those of FCDO, the UK Government, K4D or any other contributing organisation. For further information, please contact helpdesk@k4d.info. 


\section{Summary}

This literature review finds that the main malicious threats to civilian aviation since 1975 are attacks by terrorist groups, deliberate or accidental damage arising from conflicts and incidents caused by people who work for airlines or airports. While the sector has responded to hijackings and bombings with increasing security since the 1970s, actors seeking to attack aircraft have modified their tactics, and new threats such as liquid explosives and cyber attacks have emerged. Civilian aviation has seen relatively fewer accidents and deaths over the years, but threats remain.

The review focuses on malicious threats to civilian aviation. It therefore excludes weather events or accidents. ${ }^{1}$ The first section lists major malicious threats to civilian aviation since 1975. It includes both actual and planned events (e.g. hijackings that were prevented) that are recorded in open-source documents. Each threat is listed alongside information on its cause (e.g. terrorism, state actions, crime), the context in which it occurred (broader factors shaping the risk including geography, regime type, technology) and its impact (on passengers, policy, security, economic).

The second section discusses some of the trends in threats to aviation. Motives for malicious threats include terrorism, crime, asylum seeking and insider attacks by aggrieved or mentally ill airline staff. Hijacking has been the most common form of threat, although bombing or suicide attacks have killed more people. Threats may also take the form of accidental attacks on civilian planes misidentified as threats in conflict zones. Experts suggest that growing threats are cyber attacks and the use of unmanned aerial vehicles, although neither has yet caused a major incident.

The civilian aviation sector has seen increased security over the years, from passenger and baggage checks to x-ray and metal detecting scans, and guards on board, often in response to incidents. Strategies for dealing with threats to aviation centre on security measures at airports, in passenger screening, and on aeroplanes. They also include decisions on how to respond to threats, such as whether to negotiate and what measures to use in hostage situations, for example. Strategies in place to address the risk from rogue airline or airport staff include better psychometric screening and flight procedures (e.g. the number of pilots in cabin); and better job security to reduce stress and the attractiveness of bribes.

The number of incidents in the sector overall, relative to the number of flights, has decreased. ${ }^{2}$ However, incidents such as a terrorist attack, a stray missile or a disaffected crew member, remain significant threats. In addition, several authors point to airlines' and airport operators' vested interest in keeping passenger numbers high which can lead to delays in implementing security measures that delay flights.

The visibility of air travel makes it appealing to hijackers or bombers. Destroying or taking control of an aeroplane can give a group or individual significant leverage, as well as high levels of publicity. Aims vary significantly, but can include asking for ransoms, the release of political

${ }^{1}$ Sabotage only accounts for around $10 \%$ of aviation disasters, with pilot error, mechanical failures and weather much greater risks. https://theconversation.com/the-five-most-common-reasons-for-airliner-disasters-50100

${ }^{2}$ see section 2 and appendix 
prisoners, the opportunity to create a spectacle, seeking asylum, the airing of grievances, or killing particular people/groups. Aircraft are also vulnerable to accidental shooting down by armed groups or state armies. They may be used by pilots or crew members, who can bypass many security measures, to commit suicide or make an insurance claim.

Different forms of attack are more prevalent in different times and places, shaped by geopolitical conditions and security measures. For instance, hijacking was driven by decolonisation struggles and the Cold War in the 1960s and 1970s. It was dramatically reduced within the United States by increased security measures in the 1970s, but US-flagged airlines operating elsewhere remained vulnerable to hijackings. Groups or individuals who aim to use planes and airline passengers as leverage, accounting for most hijackings until the $2000 \mathrm{~s}$, present a different threat to groups who aim to maximise damage and therefore cannot be negotiated with. The threat of missile attacks is much greater in conflict zones or areas where non-state armed groups operate independently of effective command structures. In it unclear how capable airlines and airports are to deal with threats from cyber-attacks and unmanned aerial vehicle (UAVs).

\section{Incidents}

The first hijacking happened in 1931 when revolutionaries in Peru coerced an aeroplane owner to carry them. The first airline bombing occurred in 1933 on a United Airlines flight from Cleveland to Chicago and killed 7 people (Price and Forrest, 2016, p. 51). However, attacks on civilian aviation are seen to have become a significant problem by the 1960 s when terrorist groups adopted hijacking and bombing to further their ends.

Malicious incidents can be defined and categorised in a number of ways. This review includes all malicious ${ }^{3}$ activity disrupting civilian aviation in some way. It includes acts motivated by political, monetary, other, or unknown reasons. Types of incident include hijackings, bombings, suicide bombings, deliberate crashes, missile attacks, gun or knife attacks, cyber attacks and attacks by unmanned aerial vehicle (UAVs). It also includes apparently accidental attacks, such as the shooting down of planes misidentified as threats by militaries or non-state armed groups.

The list of incidents has been derived from a number of open-access sources, including the aviation safety online database, academic articles and news sources. The list is not comprehensive, and incidents are selected for their significance, understood as death toll, media attention, and effect on aviation security regimes or international relations. It omits a large number of hijackings, attempted hijackings, bombings, missile attacks and other incidents. The next section of the report discusses the trends in threats to civilian aviation and outlines emerging threats.

Figure 1: non-comprehensive list of malicious incidents featuring civilian aviation since 1975.

\footnotetext{
${ }^{3}$ While most of the incidents discussed are crimes, their precise status varies from state to state and over time as new anti-hijacking or anti-terrorism laws have been implemented. The popular understandings of many hijackings also varies significantly, particularly hijackings connected to national liberation movements such as Palestine's.
} 


\begin{tabular}{|c|c|c|c|c|}
\hline Date & Incident & Cause & Context & Impact \\
\hline 1975 & Malév flight MA240 4 & Unknown & $\begin{array}{l}\text { Possibly related to } \\
\text { disputes over } \\
\text { Palestine }^{5}\end{array}$ & 60 killed \\
\hline 1976 & $\begin{array}{l}\text { Philippine Airlines } \\
116 \text { hijacked }^{6}\end{array}$ & $\begin{array}{l}\text { Six hijackers took over } \\
\text { the plane and } \\
\text { demanded money and a } \\
\text { flight to Libya. After } \\
\text { landing to refuel, they } \\
\text { were overwhelmed by } \\
\text { security forces and } \\
\text { passengers were killed } \\
\text { in the fight. }\end{array}$ & $\begin{array}{l}\text { Islamic separatist } \\
\text { movement. }{ }^{7}\end{array}$ & 13 killed \\
\hline \multirow[t]{3}{*}{1976} & \multirow{3}{*}{$\begin{array}{l}\text { Air France flight } 139 \\
\text { hijacked }^{8}\end{array}$} & \multirow{3}{*}{$\begin{array}{l}\text { Members of the Baader- } \\
\text { Meinhof gang hijacked a } \\
\text { plane and took it to } \\
\text { Entebbe, Uganda. They } \\
\text { released all except the } \\
105 \text { Israeli and Jewish } \\
\text { passengers, and } \\
\text { demanded Israel } \\
\text { release } 53 \text { Palestinian } \\
\text { prisoners. The hijackers } \\
\text { were overcome by } \\
\text { Israeli commandos. }\end{array}$} & Left-wing terrorism. & 3 hostages and killed. \\
\hline & & & $\begin{array}{l}\text { Israel-Palestine } \\
\text { conflict. }\end{array}$ & $\begin{array}{l}\text { Increased security at } \\
\text { Israeli airports and } \\
\text { airlines. }\end{array}$ \\
\hline & & & & $\begin{array}{l}\text { Hardened no- } \\
\text { negotiation stance. }\end{array}$ \\
\hline 1976 & $\begin{array}{l}\text { Cubana de Aviación } \\
455 \text { bombed }^{9}\end{array}$ & $\begin{array}{l}\text { Venezuela blames US- } \\
\text { backed Cubans }{ }^{10}\end{array}$ & $\begin{array}{l}\text { Disputes between the } \\
\text { US and communist } \\
\text { Cuba }\end{array}$ & 73 killed \\
\hline 1976 & $\begin{array}{l}\text { Middle East Airlines } \\
438 \text { exploded over } \\
\text { the Saudi Arabian }\end{array}$ & $\begin{array}{l}\text { Bomb in the baggage } \\
\text { hold. }\end{array}$ & $\begin{array}{l}\text { Bombers not identified } \\
\text { by possibly related to }\end{array}$ & 81 killed \\
\hline
\end{tabular}

\footnotetext{
${ }^{4}$ https://aviation-safety.net/database/record.php?id=19750930-1

${ }^{5}$ https://aeronauticsonline.com/still-no-hope-in-the-most-mysterious-crash/

${ }^{6}$ https://aviation-safety.net/database/record.php?id=19760523-1

7 https://www.nytimes.com/1976/05/24/archives/3-hijackers-and-10-hostages-die-as-philippine-plane-is-setafire.html

${ }^{8}$ https://aviation-safety.net/database/record.php?id=19760627-1

${ }^{9}$ https://aviation-safety.net/database/record.php?id=19761006-0

${ }^{10}$ http://news.bbc.co.uk/1/hi/world/americas/4289136.stm
} 


\begin{tabular}{|c|c|c|c|c|}
\hline & desert $^{11}$ & & conflict in Oman ${ }^{12}$ & \\
\hline 1977 & $\begin{array}{l}\text { Malaysian Airlines } \\
\text { System flight } 653 \\
\text { hijacked and } \\
\text { crashed }^{13}\end{array}$ & $\begin{array}{l}\text { Hijackers demanded to } \\
\text { be flown to Singapore, } \\
\text { then shot pilots and } \\
\text { themselves. }\end{array}$ & $\begin{array}{l}\text { Hijackers have never } \\
\text { been identified }^{14}\end{array}$ & 100 killed \\
\hline 1977 & $\begin{array}{l}\text { Lufthansa flight } 181 \\
\text { hijacked }\end{array}$ & $\begin{array}{l}\text { Popular Front for the } \\
\text { Liberation of Palestine } \\
\text { members demanded } \\
\text { released of Red Army } \\
\text { Faction prisoners and } \\
\text { USD } 15 \text { million. The } \\
\text { plane eventually landed } \\
\text { in Mogadishu where it } \\
\text { was stormed by West } \\
\text { German and Somali } \\
\text { commandos. }\end{array}$ & $\begin{array}{l}\text { Israel-Palestinian } \\
\text { conflict. } \\
\text { RAF campaign. }\end{array}$ & $\begin{array}{l}4 \text { killed. } \\
\text { West Germany's non- } \\
\text { negotiation stance } \\
\text { developed after } \\
\text { previous hijackings } \\
\text { reaffirmed. } \\
\text { Improved flight links } \\
\text { between West } \\
\text { Germany and } \\
\text { Somalia. }\end{array}$ \\
\hline 1977 & $\begin{array}{l}\text { Japan Air Lines flight } \\
472 \text { hijacked }^{15}\end{array}$ & $\begin{array}{l}\text { Five armed Japanese } \\
\text { Red Army (JRA) } \\
\text { members demanded the } \\
\text { release of political } \\
\text { prisoners and USD } 6 \\
\text { million. } \\
\text { The Japanese } \\
\text { government acquiesced } \\
\text { and all the hostages } \\
\text { were released }\end{array}$ & Left wing terrorism & $\begin{array}{l}0 \text { killed } \\
\text { Establishment of a } \\
\text { Special Assault Team } \\
\text { within the Japanese } \\
\text { police to deal with } \\
\text { hijackings. }\end{array}$ \\
\hline 1978 & $\begin{array}{l}\text { Shooting down of } \\
\text { Korean Air Lines } \\
\text { Flight } 902^{16}\end{array}$ & $\begin{array}{l}\text { Navigation errors. Was } \\
\text { shot down by Soviet } \\
\text { fighter jets after entering } \\
\text { Soviet airspace. }\end{array}$ & Cold War & 2 passengers died. \\
\hline 1978 & $\begin{array}{l}\text { Air Rhodesia Flight } \\
825^{17}\end{array}$ & $\begin{array}{l}\text { Shot down with a Strela } \\
2 \text { surface-to-air missile } \\
\text { by the Zimbabwe } \\
\text { People's Revolutionary } \\
\text { Army. }\end{array}$ & $\begin{array}{l}\text { Conflict between } \\
\text { colonial and anti- } \\
\text { colonial forces. }\end{array}$ & $\begin{array}{l}38 \text { passengers died } \\
\text { in the crash and } 10 \\
\text { were killed by } \\
\text { guerrillas after the } \\
\text { crash. } \\
\text { Increased racial }\end{array}$ \\
\hline
\end{tabular}

\footnotetext{
${ }^{11}$ https://aviation-safety.net/database/record.php?id=19760101-1

12 https://news.google.com/newspapers?id=ur0yAAAAIBAJ\&sjid=qe0FAAAAIBAJ\&pg=4271\%2C4951988

${ }^{13}$ https://aviation-safety.net/database/record.php?id=19771204-0

${ }^{14}$ https://edition.cnn.com/2014/03/27/world/asia/malaysia-mh653-crash-relatives/index.html?hpt=hp_c1

15 https://aviation-safety.net/database/record.php?id=19770928-0

${ }^{16}$ https://aviation-safety.net/database/record.php?id=19780420-1

${ }^{17}$ https://aviation-safety.net/database/record.php?id=19780903-1
} 
tensions and vigilante violence in Zimbabwe and breakdown of peace talks.

\begin{tabular}{|c|c|c|c|c|}
\hline 1979 & $\begin{array}{l}\text { Air Rhodesia Flight } \\
827^{18}\end{array}$ & $\begin{array}{l}\text { Shot down with a Strela } \\
2 \text { surface-to-air missile } \\
\text { by the Zimbabwe } \\
\text { People's Revolutionary } \\
\text { Army. }\end{array}$ & $\begin{array}{l}\text { Conflict between } \\
\text { colonial and anti- } \\
\text { colonial forces. Attempt } \\
\text { to assassinate military } \\
\text { figure. }\end{array}$ & $\begin{array}{l}59 \text { killed. Escalation } \\
\text { of conflict. } \\
\text { Low radiation paint to } \\
\text { shroud exhaust } \\
\text { fumes and make } \\
\text { planes less visible }\end{array}$ \\
\hline 1979 & $\begin{array}{l}\text { United Airlines flight } \\
444 \text { bombed }^{19}\end{array}$ & $\begin{array}{l}\text { A bomb with a } \\
\text { barometric trigger } \\
\text { exploded in the baggage } \\
\text { hold, but the plane was } \\
\text { landed safely }\end{array}$ & $\begin{array}{l}\text { Traced to Ted } \\
\text { Kaczynski, the } \\
\text { 'Unabomber', a lone } \\
\text { terrorist }\end{array}$ & 0 killed \\
\hline 1980 & $\begin{array}{l}\text { Explosion on Itavia } \\
\text { Flight } 870 \text { from } \\
\text { Bologna to Palermo }{ }^{20}\end{array}$ & $\begin{array}{l}\text { Unknown. Perhaps } \\
\text { accidental fire from a } \\
\text { NATO exercise, or a } \\
\text { bomb on the plane. }\end{array}$ & & 81 killed \\
\hline 1980 & $\begin{array}{l}\text { Crash of Linhas } \\
\text { Aéreas de Angola } \\
\text { Yakovlev Yak-4021 }\end{array}$ & Possibly shot down & Angolan civil war & 13 killed \\
\hline 1982 & $\begin{array}{l}\text { Pan Am Flight } 830 \\
\text { was bombed } \\
\text { between Tokyo and } \\
\text { Hawaii }^{22}\end{array}$ & $\begin{array}{l}\text { Is alleged to have been } \\
\text { placed by Mohammed } \\
\text { Rashed, a Jordanian } \\
\text { connected to the } 15 \text { May } \\
\text { Organization, a small } \\
\text { Palestinian liberation } \\
\text { group. }\end{array}$ & $\begin{array}{l}\text { Israel-Palestine } \\
\text { conflict. }\end{array}$ & 1 killed \\
\hline 1982 & $\begin{array}{l}\text { Japan Airlines flight } \\
350\end{array}$ & $\begin{array}{l}\text { Pilot deliberately } \\
\text { crashed the plane }\end{array}$ & $\begin{array}{l}\text { Mental health and job } \\
\text { insecurity (Nori, 2020, } \\
\text { p. 63) }\end{array}$ & 24 killed \\
\hline 1983 & $\begin{array}{l}\text { Korean Air Lines } \\
\text { Flight } 007^{23}\end{array}$ & $\begin{array}{l}\text { Navigation errors. Was } \\
\text { shot down by Soviet }\end{array}$ & Cold War & $\begin{array}{l}269 \text { killed. } \\
\text { Increased Cold War }\end{array}$ \\
\hline
\end{tabular}

\footnotetext{
18 https://aviation-safety.net/database/record.php?id=19790212-1

${ }^{19}$ https://aviation-safety.net/database/record.php?id=19791115-1

${ }^{20}$ https://aviation-safety.net/database/record.php?id=19800627-0

${ }^{21}$ https://aviation-safety.net/database/record.php?id=19800608-0

22 https://aviation-safety.net/database/record.php?id=19820811-0

${ }^{23}$ https://aviation-safety.net/database/record.php?id=19830901-0
} 


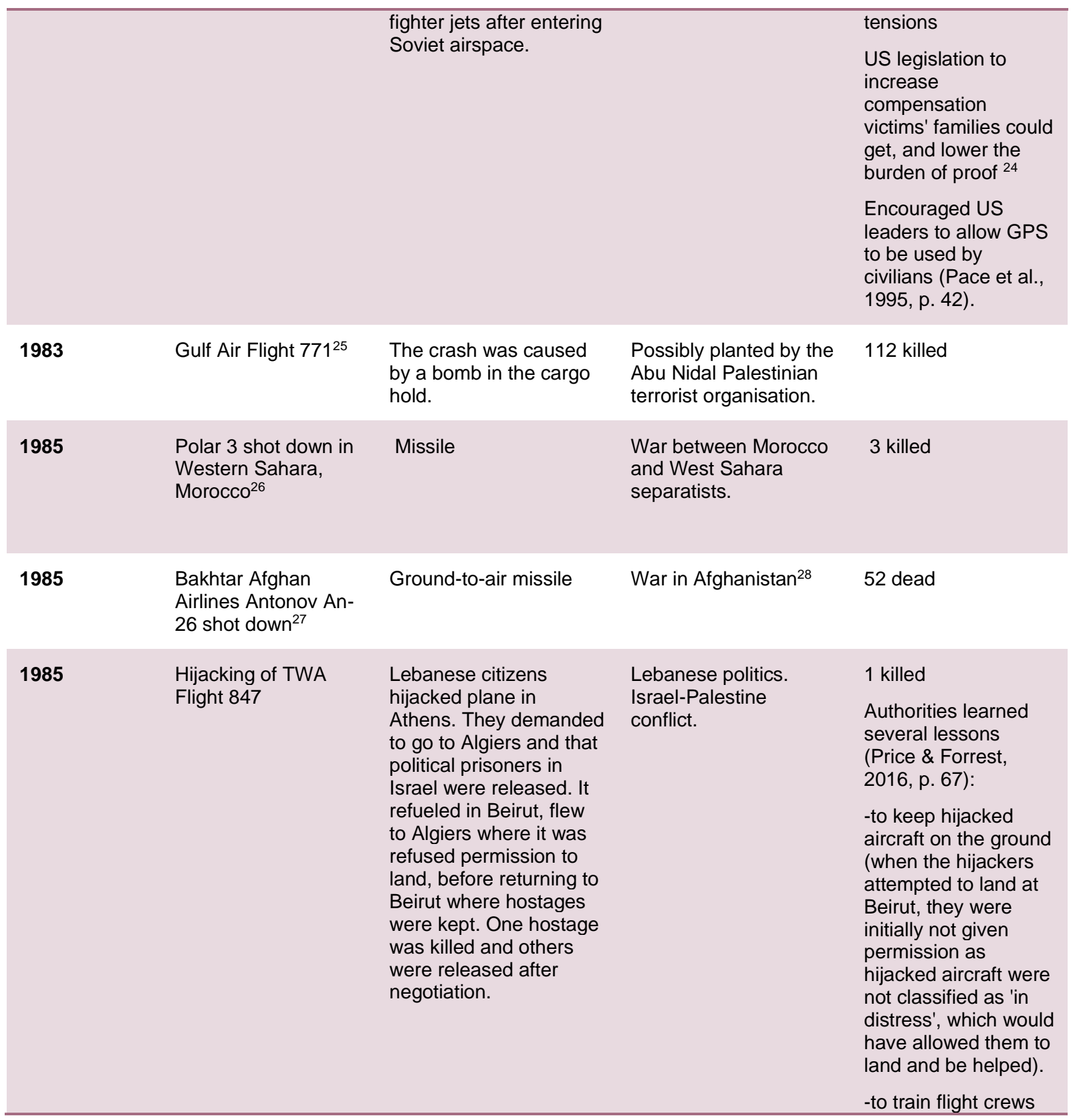

24 https://www.nytimes.com/1997/03/31/nyregion/grieving-father-s-14-year-crusade-helps-air-crashvictims.html?auth=login-google

${ }^{25}$ gulf air 8

${ }^{26}$ https://aviation-safety.net/database/record.php?id=19850224-0

27 https://aviation-safety.net/database/record.php?id=19850904-0

28 https://www.nytimes.com/1985/09/09/world/afghan-rebels-deny-kabul-report-that-they-downed-civilairlines.html 


\begin{tabular}{|c|c|c|c|c|}
\hline & & & & $\begin{array}{l}\text { in emergency safety } \\
\text { and crisis } \\
\text { management. }\end{array}$ \\
\hline \multirow[t]{3}{*}{1985} & \multirow[t]{3}{*}{ Air India $181 / 182^{29}$} & \multirow{3}{*}{$\begin{array}{l}\text { A bomb exploded on a } \\
\text { flight between Montreal } \\
\text { and London. } \\
\text { It may be linked with an } \\
\text { explosion the same day } \\
\text { at Tokyo-Narita airport. }\end{array}$} & \multirow{3}{*}{$\begin{array}{l}\text { Possibly extremist Sikh } \\
\text { group Babbar Khalsa }{ }^{30} \\
\text { (Price and Forrest, } \\
2016 \text { p. 68) }\end{array}$} & \multirow{2}{*}{$\begin{array}{l}329 \text { killed } \\
\text { A five step screening } \\
\text { process (including x- } \\
\text { ray, visual, } \\
\text { explosives) was } \\
\text { instituted in Canada } \\
\text { (Price \& Forrest, } \\
2016, \text { p. 69) }\end{array}$} \\
\hline & & & & \\
\hline & & & & $\begin{array}{l}\text { A Canadian } \\
\text { government enquiry } \\
\text { was finished in } 2010 \\
\text { and concluded, } \\
\text { among other things, } \\
\text { that security flaws } \\
\text { remained, particularly } \\
\text { in respect to cargo }{ }^{31}\end{array}$ \\
\hline \multirow[t]{2}{*}{1985} & \multirow{2}{*}{$\begin{array}{l}\text { Egypt Air } 648 \\
\text { hijacked and } 61 \text { killed } \\
\text { from shooting by the } \\
\text { hijackers and when } \\
\text { Egyptian forces } \\
\text { stormed the plane in } \\
\text { Malta }^{32}\end{array}$} & \multirow{2}{*}{$\begin{array}{l}\text { Members of the Abu } \\
\text { Nidal organisation }\end{array}$} & \multirow{2}{*}{$\begin{array}{l}\text { Israel-Palestine } \\
\text { conflict. }\end{array}$} & 61 killed \\
\hline & & & & $\begin{array}{l}\text { Criticism of the } \\
\text { Maltese response } \\
\text { and the Egyptian } \\
\text { operation }^{33}\end{array}$ \\
\hline 1985 & $\begin{array}{l}\text { Rome Airport } \\
\text { attacked by men with } \\
\text { guns and grenades }\end{array}$ & $\begin{array}{l}\text { Members of the Abu } \\
\text { NIdal Organisation } \\
\text { (ANO) (Duchesneau \& } \\
\text { Langlois, 2017, p. 347) }\end{array}$ & Israel-Palestine conflict & $\begin{array}{l}16 \text { passengers and } 3 \\
\text { gunmen killed }\end{array}$ \\
\hline 1985 & $\begin{array}{l}\text { Vienna Airport attack } \\
\text { with grenades }\end{array}$ & $\begin{array}{l}\text { Members of the Abu } \\
\text { Nidal Organisation } \\
\text { (ANO) }\end{array}$ & Israel-Palestine conflict & 3 passengers killed \\
\hline 1986 & TWA Flight $840^{34}$ & $\begin{array}{l}\text { A bomb exploded on a } \\
\text { flight between Rome } \\
\text { and Athens }\end{array}$ & $\begin{array}{l}\text { A member of the Abu } \\
\text { Nidal extremist group } \\
\text { was suspected but no } \\
\text { one has been }\end{array}$ & 4 killed \\
\hline
\end{tabular}

\footnotetext{
${ }^{29}$ https://aviation-safety.net/database/record.php?id=19850623-2

30 http://news.bbc.co.uk/1/hi/world/americas/4344051.stm

31 https://epe.lac-bac.gc.ca/100/206/301/pco-bcp/commissions/air_india/2010-07-

23/www.majorcomm.ca/en/reports/finalreport/key-findings.pdf

32 https://aviation-safety.net/database/record.php?id=19851124-0

${ }^{33}$ http://news.bbc.co.uk/onthisday/hi/dates/stories/november/24/newsid_4356000/4356024.stm

${ }^{34}$ https://aviation-safety.net/database/record.php?id=19860402-1
} 


\begin{tabular}{|c|c|c|c|c|}
\hline 1986 & El Al Flight 016 & $\begin{array}{l}\text { Nezar Hindawi was } \\
\text { found guilty of planning } \\
\text { to bomb the plane by } \\
\text { putting explosives in his } \\
\text { fiancé's luggage. }\end{array}$ & & $\begin{array}{l}0 \\
\text { Diplomatic dispute } \\
\text { between West and } \\
\text { Syria. } \\
\text { Raised the issue of } \\
\text { unwitting bomb } \\
\text { carriers. }\end{array}$ \\
\hline 1986 & $\begin{array}{l}\text { Air Lanka Flight } \\
512^{36}\end{array}$ & $\begin{array}{l}\text { A bomb exploded during } \\
\text { loading }\end{array}$ & Sri Lankan civil war & 14 killed \\
\hline 1986 & $\begin{array}{l}\text { Iraqi Airways Flight } \\
163^{37}\end{array}$ & $\begin{array}{l}\text { Hijackers exploded } \\
\text { grenades during the } \\
\text { flight. Hezbollah claimed } \\
\text { responsibility. }\end{array}$ & $\begin{array}{l}\text { Terrorism; Middle East } \\
\text { tensions }\end{array}$ & 63 killed \\
\hline 1986 & China Airlines 334 & $\begin{array}{l}\text { Pilot hijacked a freight } \\
\text { plane from Taiwan to } \\
\text { Thailand and took it to } \\
\text { communist China. } \\
\text { Possibly politically } \\
\text { driven (defection to } \\
\text { communist China) or to } \\
\text { reunite with father in } \\
\text { Sichuan (Nori, 2020, p. } \\
65 \text { ) }\end{array}$ & $\begin{array}{l}\text { Cold War } \\
\text { ideologies/family } \\
\text { reunion. }\end{array}$ & $\begin{array}{l}0 \text { killed. } \\
\text { China returned the } \\
\text { plane to Taiwan. This } \\
\text { was the first meeting } \\
\text { of Taiwanese and } \\
\text { Chinese officials } \\
\text { since 1949, and } \\
\text { contributed to a thaw } \\
\text { in relations. }\end{array}$ \\
\hline 1986 & $\begin{array}{l}\text { Pan Am flight } 73 \\
\text { hijacked }\end{array}$ & $\begin{array}{l}\text { Hijackers boarded the } \\
\text { plane disguised as } \\
\text { security guards. }^{39}\end{array}$ & $\begin{array}{l}\text { Possibly seeking the } \\
\text { release of political } \\
\text { prisoners in Cyprus }{ }^{40}\end{array}$ & 20 killed \\
\hline 1986 & $\begin{array}{l}\text { Sudan Airways flight } \\
\text { hit by a missile }\end{array}$ & $\begin{array}{l}\text { Surface-to-air missile } \\
\text { fired by the Sudan } \\
\text { People's Liberation } \\
\text { Army. }\end{array}$ & Sudanese civil war & 60 killed \\
\hline 1987 & $\begin{array}{l}\text { Downing of PSA } \\
\text { Flight } 1771 \text {, }\end{array}$ & $\begin{array}{l}\text { A disgruntled ex- } \\
\text { employee killed his } \\
\text { former supervisor, flight } \\
\text { crew, and himself, }\end{array}$ & Personal/employment. & $\begin{array}{l}43 \text { killed } \\
\text { US law changes to } \\
\text { ensure that airline }\end{array}$ \\
\hline
\end{tabular}

\footnotetext{
35 http://news.bbc.co.uk/onthisday/hi/dates/stories/april/2/newsid_4357000/4357159.stm

${ }^{36}$ https://aviation-safety.net/database/record.php?id=19860503-1

${ }^{37}$ https://aviation-safety.net/database/record.php?id=19861225-1

38 https://www.nytimes.com/1986/05/24/world/china-returns-hijacked-jet-and-2-crewmen-to-taiwan.html

${ }^{39}$ http://news.bbc.co.uk/onthisday/hi/dates/stories/september/5/newsid_4576000/4576765.stm

40 https://aviation-safety.net/database/record.php?id=19860905-0
} 


\begin{tabular}{|c|c|c|c|c|}
\hline & & $\begin{array}{l}\text { causing the plane to } \\
\text { crash and killing } 54 \text {. He } \\
\text { had used his staff pass } \\
\text { to bypass security (Nori, } \\
\text { 2020; Price \& Forrest, } \\
\text { 2016). }\end{array}$ & & $\begin{array}{l}\text { employees hand in } \\
\text { security passes as } \\
\text { soon as they leave } \\
\text { employment. } \\
\text { Many large } \\
\text { companies began to } \\
\text { insist that their } \\
\text { executives travelled } \\
\text { on separate flights, } \\
\text { after several lost } \\
\text { more than one senior } \\
\text { manager in this } \\
\text { flight. }{ }^{41}\end{array}$ \\
\hline 1987 & $\begin{array}{l}\text { Bakhtar Afghan } \\
\text { Airlines Antonov An- } \\
26^{42}\end{array}$ & Ground-to-air missile & War in Afghanistan & 53 dead \\
\hline 1987 & $\begin{array}{l}\text { Zimex Aviation } \\
\text { Lockheed L-100, } \\
\text { Angola }^{43}\end{array}$ & $\begin{array}{l}\text { A Red Cross plane was } \\
\text { hit by a missile, which } \\
\text { may have been fired by } \\
\text { a conflict party }{ }^{4}\end{array}$ & Angolan civil war & 6 killed \\
\hline 1987 & Air Malawi 7Q-YMB ${ }^{45}$ & $\begin{array}{l}\text { A domestic flight in } \\
\text { Malawi was shot down } \\
\text { over Mozambique }\end{array}$ & Mozambique civil war & 10 killed \\
\hline 1987 & $\begin{array}{l}\text { Korean Air Flight } 858 \\
\text { was destroyed in } \\
\text { mid-air }^{46}\end{array}$ & $\begin{array}{l}\text { C-4 explosives placed } \\
\text { by two North Koreans. }\end{array}$ & $\begin{array}{l}\text { Conflict between North } \\
\text { and South Korea }\end{array}$ & $\begin{array}{l}115 \text { killed. Continued } \\
\text { tensions. } \\
\text { North Korea placed } \\
\text { on the US list of state } \\
\text { sponsors of terrorism. }\end{array}$ \\
\hline 1988 & $\begin{array}{l}\text { Iran Air Flight } 655 \text { hit } \\
\text { by missile } 47\end{array}$ & $\begin{array}{l}\text { Missiles fired by the US } \\
\text { Navy when misidentified } \\
\text { as a military threat, } \\
\text { possibly due to tensions }\end{array}$ & Iran-Iraq war. & $\begin{array}{l}290 \text { killed } \\
\text { Increased US-Iran }\end{array}$ \\
\hline
\end{tabular}

\footnotetext{
${ }^{41}$ https://slate.com/news-and-politics/2010/04/poland-s-leading-political-figures-died-in-a-plane-crash-over-theweekend-could-that-happen-here.html

42 https://aviation-safety.net/database/record.php?id=19870611-0

43 https://aviation-safety.net/database/record.php?id=19871014-0

44 https://apnews.com/article/a79c31b3580287c8e13df7b00bf87cf2

${ }^{45}$ https://aviation-safety.net/database/record.php?id=19871106-1

${ }^{46}$ https://aviation-safety.net/database/record.php?id=19871129-0

${ }^{47}$ https://aviation-safety.net/database/record.php?id=19880703-0
} 
around the war,

software flaws and radio

communication failures. tensions.

US paid

compensation but did

not acknowledge

fault.

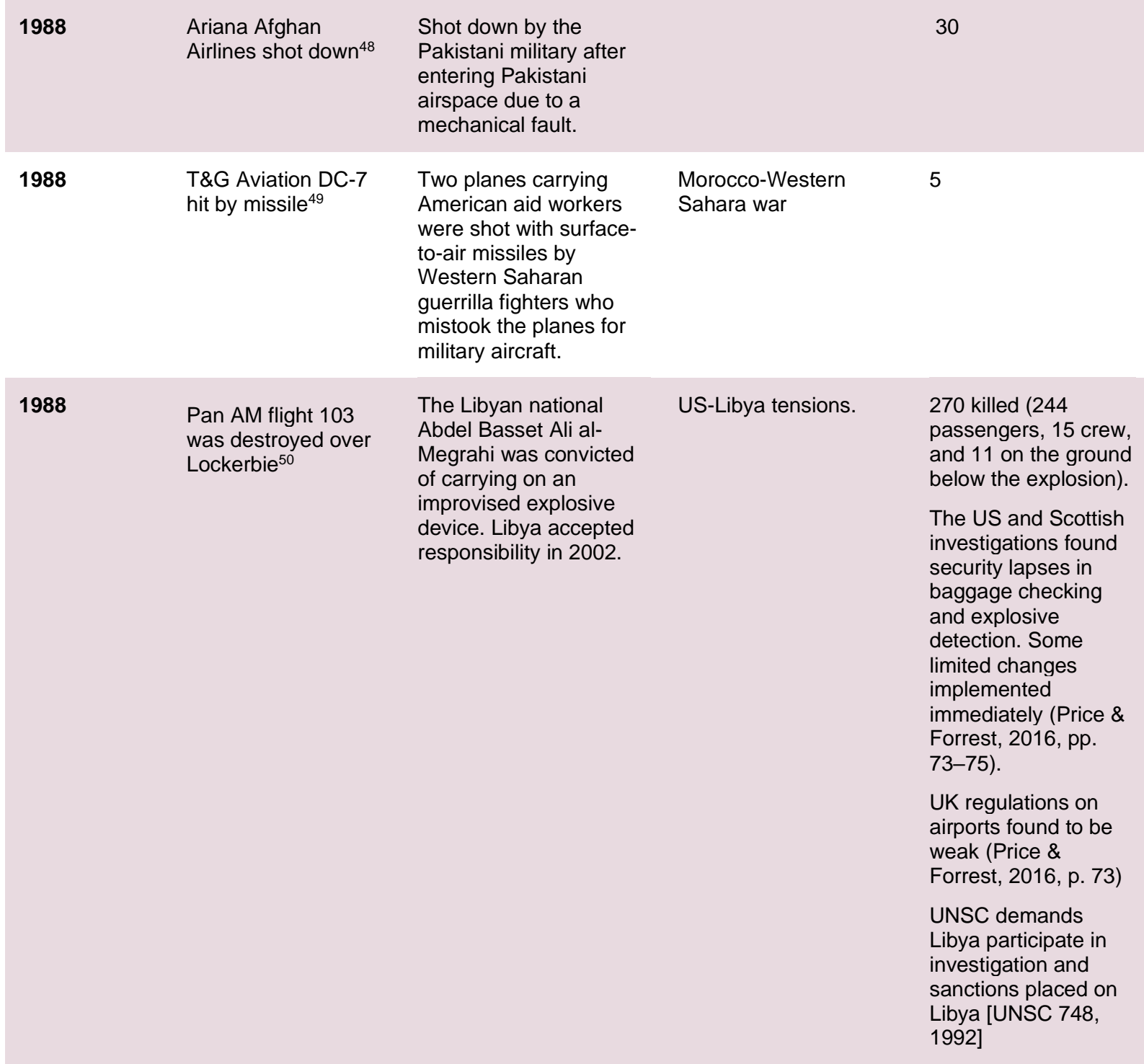

\footnotetext{
48 https://aviation-safety.net/database/record.php?id=19881119-0

49 https://aviation-safety.net/database/record.php?id=19881208-1

${ }^{50}$ https://aviation-safety.net/database/record.php?id=19881221-0
} 


\begin{tabular}{|c|c|c|c|c|}
\hline 1988 & $\begin{array}{l}\text { Aeroflot flight } 3729 \\
\text { hijacked }^{52}\end{array}$ & $\begin{array}{l}\text { A family of } 11 \text { hijacked } \\
\text { the plane and asked to } \\
\text { be flown to London. } \\
\text { When refuelling in } \\
\text { Leningrad, Soviet troops } \\
\text { stormed the plane. }\end{array}$ & Soviet policies/asylum. & 9 killed \\
\hline 1989 & $\begin{array}{l}\text { UTA Flight } 772 \text { was } \\
\text { destroyed by a } \\
\text { bomb }^{53}\end{array}$ & $\begin{array}{l}\text { Six Libyans were } \\
\text { convicted }\end{array}$ & Chad-Libyan war & 170 killed \\
\hline 1989 & Avianca Flight $203^{54}$ & $\begin{array}{l}\text { An improvised explosive } \\
\text { device placed by a } \\
\text { member of the Medellin } \\
\text { drug cartel caused the } \\
\text { plane to break up, } \\
\text { possibly to kill informers. }\end{array}$ & $\begin{array}{l}\text { Conflict between the } \\
\text { Medellin drug cartel, } \\
\text { the Colombian state } \\
\text { and the US. }\end{array}$ & $\begin{array}{l}107 \text { killed } \\
\text { US action against } \\
\text { Pablo Escobar, } \\
\text { leader of the Medellin } \\
\text { cartel. }\end{array}$ \\
\hline 1990 & $\begin{array}{l}\text { Xiamen Airlines } 8301 \\
\text { hijacked }^{55}\end{array}$ & $\begin{array}{l}\text { A man hijacked a } \\
\text { Chinese domestic flight } \\
\text { and asked to be taken to } \\
\text { Taiwan so he could } \\
\text { request asylum. He had } \\
\text { also stolen money from } \\
\text { his company (Nori, } \\
2020, \text { p. 67) }\end{array}$ & $\begin{array}{l}\text { Political conditions in } \\
\text { China; personal } \\
\text { reasons. }\end{array}$ & $\begin{array}{l}84 \text { killed on the plane } \\
\text { and } 47 \text { killed on a } \\
\text { plane it hit when the } \\
\text { hijacker took control. }\end{array}$ \\
\hline 1993 & $\begin{array}{l}\text { Transair Georgia and } \\
\text { Orbia Georgian } \\
\text { airways planes shot } \\
\text { down }\end{array}$ & $\begin{array}{l}\text { Shot by a surface-to-air } \\
\text { missile by Abkhazian } \\
\text { separatists }\end{array}$ & War in Abkhazia. & 136 killed \\
\hline 1994 & $\begin{array}{l}\text { Rwandan } \\
\text { government plane } \\
\text { shot down }\end{array}$ & $\begin{array}{l}\text { Surface-to-air missiles } \\
\text { fired by elements } \\
\text { seeking to disrupt power } \\
\text { sharing between Hutus } \\
\text { and Tutsis }\end{array}$ & $\begin{array}{l}\text { Hutu-Tutsi tensions in } \\
\text { the Great Lakes region. }\end{array}$ & $\begin{array}{l}12 \text { killed, including } \\
\text { Rwandan and } \\
\text { Burundian } \\
\text { presidents. } \\
\text { A trigger for conflict } \\
\text { and genocide. }\end{array}$ \\
\hline
\end{tabular}

\footnotetext{
51 https://www.propublica.org/article/20-years-after-lockerbie-aviation-security-gaps-remain-1222; https://www.nytimes.com/2001/01/31/world/long-before-verdict-lockerbie-changed-airportsecurity.html\#: :text=The\%20system\%20does\%20not\%20require,sniffer\%20machines\%20or\%20other\%20mean

52 https://aviation-safety.net/database/record.php?id=19880308-0

${ }^{53}$ https://aviation-safety.net/database/record.php?id=19890919-1

54 https://aviation-safety.net/database/record.php?id=19891127-0

${ }^{55}$ https://aviation-safety.net/database/record.php?id=19901002-3

${ }^{56}$ https://aviation-safety.net/database/record.php?id=19940406-1
} 


\begin{tabular}{|c|c|c|c|c|}
\hline 1994 & $\begin{array}{l}\text { Philippine Airlines } \\
\text { Flight } 434 \text { was hit by } \\
\text { an improvised } \\
\text { explosive device. }{ }^{57}\end{array}$ & $\begin{array}{l}\text { Ramzi Yousef, an } \\
\text { Islamic terrorist, } \\
\text { assembled the bomb in } \\
\text { the plane toilet before } \\
\text { leaving it under a seat } \\
\text { and got off the plane. } \\
\text { The bomb killed one } \\
\text { passenger. }\end{array}$ & Islamic terrorism & $\begin{array}{l}1 \text { killed. } \\
\text { Yousef was also part } \\
\text { of the unsuccessful } \\
\text { 'Bojinka plot' to } \\
\text { assassinate the Pope } \\
\text { and destroy } 11 \\
\text { passenger planes. } \\
\text { Increased security in } \\
\text { US airports - a 300- } \\
\text { foot zone around } \\
\text { airport terminals and } \\
\text { air traffic control } \\
\text { facilities where } \\
\text { vehicles were not } \\
\text { allowed to park. } \\
\text { Airports lost revenue } \\
\text { from parking (Klenka, } \\
2019, \text { p. } 47 \text { ). }\end{array}$ \\
\hline 1994 & $\begin{array}{l}\text { FedEx flight } 1994 \\
\text { hijacked }\end{array}$ & $\begin{array}{l}\text { An employee brought a } \\
\text { spear gun, hunting knife } \\
\text { and two hammers on } \\
\text { board, in the hope of his } \\
\text { family getting a life } \\
\text { insurance pay out. } \\
\text { Overpowered by crew } \\
\text { (Nori, 2020, p. 69) }\end{array}$ & $\begin{array}{l}\text { Personal factors (the } \\
\text { hijacker was likely to } \\
\text { lose his job after lying } \\
\text { about his flying } \\
\text { experience) }\end{array}$ & 0 killed \\
\hline 1994 & $\begin{array}{l}\text { Royal Air Maroc flight } \\
630 \text { crashed by pilot }\end{array}$ & $\begin{array}{l}\text { Crashed by pilot for } \\
\text { personal motives Nori, } \\
2020 \text {, p. } 69 \text { ) }\end{array}$ & Personal motives & 44 killed \\
\hline 1995 & $\begin{array}{l}\text { Kish Air flight } 707 \\
\text { hijacked by a crew } \\
\text { member }\end{array}$ & $\begin{array}{l}\text { Desire to apply for } \\
\text { asylum in Europe (Nori, } \\
2020, \text { p. 70). }\end{array}$ & Repression in Iran & 0 killed \\
\hline 1996 & $\begin{array}{l}\text { TWA flight } 800 \\
\text { crashed off New York }\end{array}$ & $\begin{array}{l}\text { Accident, although } \\
\text { feared to be a bomb or } \\
\text { missile at the time. }\end{array}$ & & $\begin{array}{l}230 \text { killed } \\
\text { The 'Gore } \\
\text { Commission' on } \\
\text { aircraft safety made } \\
\text { recommendations to } \\
\text { improve aviation } \\
\text { security }\end{array}$ \\
\hline 1996 & $\begin{array}{l}\text { Ethiopian Airlines } \\
961 \text { hijacked }\end{array}$ & $\begin{array}{l}\text { Three Ethiopians aimed } \\
\text { to claim asylum in } \\
\text { Australia. After ignoring } \\
\text { the pilot's pleas to land } \\
\text { and refuel, the plane ran } \\
\text { out of fuel and crashed. } \\
\text { The refusals to refuel } \\
\text { suggest the hijackers } \\
\text { wanted to crash the } \\
\text { plane, or were too } \\
\text { intoxicated to } \\
\text { understand. The plane }\end{array}$ & $\begin{array}{l}\text { Possibly seeking } \\
\text { asylum }\end{array}$ & 125 \\
\hline
\end{tabular}

\footnotetext{
${ }^{57}$ https://aviation-safety.net/database/record.php?id=19941211-5
} 


\begin{tabular}{|c|c|c|c|c|}
\hline & & $\begin{array}{l}\text { crash-landed and some } \\
\text { passengers survived. }{ }^{58}\end{array}$ & & \\
\hline 1997 & $\begin{array}{l}\text { Silk Air flight } 185 \\
\text { crashed by the pilot }\end{array}$ & $\begin{array}{l}\text { Suicide (Nori, 2020, p. } \\
\text { 72). }\end{array}$ & Personal reasons & 104 killed \\
\hline 1998 & Lionair Flight $602^{59}$ & $\begin{array}{l}\text { Internal Sri Lankan flight } \\
\text { shot down. }\end{array}$ & $\begin{array}{l}\text { Sri Lankan civil war. } \\
\text { Possibly shot down by } \\
\text { Liberation Tigers of } \\
\text { Tamil Eelam (LTTE) }\end{array}$ & 55 killed \\
\hline 1998 & $\begin{array}{l}\text { Lignes Aeriennes } \\
\text { Congolaises shot } \\
\text { down } 60\end{array}$ & $\begin{array}{l}\text { Surface-to-air missile } \\
\text { (SA7) }\end{array}$ & Civil war & 41 killed \\
\hline 1999 & $\begin{array}{l}\text { Air Botswana } \\
\text { aeroplane crashed } \\
\text { by pilot }\end{array}$ & $\begin{array}{l}\text { Pilot flew an empty } \\
\text { plane until it ran out of } \\
\text { fuel and crashed. } \\
\text { Personal and } \\
\text { professional reasons } \\
\text { (Nori, 2020, p. 73). }\end{array}$ & Personal reasons & 1 killed \\
\hline 1999 & $\begin{array}{l}\text { Egypt Air flight } 990 \\
\text { crashed by co-pilot }\end{array}$ & Personal reasons & $\begin{array}{l}\text { Insecurity in Egyptian } \\
\text { pilot profession as a } \\
\text { possible contributing } \\
\text { factor (Nori, 2020, p. } \\
77 \text { ). }\end{array}$ & 217 killed \\
\hline 2001 & $\begin{array}{l}\text { Siberian Airlines } \\
\text { flight } 1812 \text { from } \\
\text { Israel to Russia was } \\
\text { shot down by } \\
\text { Ukrainian defence } \\
\text { force S-200V } \\
\text { missiles }^{61}\end{array}$ & $\begin{array}{l}\text { Shot by Ukrainian } \\
\text { defence force S- } 200 \mathrm{~V} \\
\text { missiles by accident, }\end{array}$ & Military manoeuvres. & 78 killed. \\
\hline 2001 & $\begin{array}{l}\text { Cessna A185E shot } \\
\text { down by Peruvian Air } \\
\text { Force aided by CIA. }\end{array}$ & $\begin{array}{l}\text { The CIA misidentified } \\
\text { the plane as a potential } \\
\text { target. }\end{array}$ & War on Drugs & $\begin{array}{l}2 \text { killed. } \\
\text { CIA amended } \\
\text { practices }\end{array}$ \\
\hline 2001 & $\begin{array}{l}\text { Attacks on the US } \\
\text { World Trade Center } \\
\text { and Pentagon }\end{array}$ & $\begin{array}{l}\text { Al-Qaeda members } \\
\text { hijacked } 4 \text { planes, } \\
\text { crashing } 2 \text { into the } \\
\text { World Trade Center in } \\
\text { New York, one into the } \\
\text { Pentagon, and one } \\
\text { crashed into a field as } \\
\text { the hijacker was } \\
\text { overpowered. }\end{array}$ & Islamic extremism & $\begin{array}{l}2,996 \text { killed } \\
\text { Increased security at } \\
\text { airports around the } \\
\text { world. } \\
\text { Increased security on } \\
\text { board including } \\
\text { armoured flight deck } \\
\text { doors. } \\
\text { Most hijackings since } \\
\text { then have been } \\
\text { thwarted (Price \& } \\
\text { Forrest, 2016, p. 88) }\end{array}$ \\
\hline
\end{tabular}

\footnotetext{
${ }^{58}$ https://aviation-safety.net/database/record.php?id=19961123-0

${ }^{59}$ https://aviation-safety.net/database/record.php?id=19980929-0

60 https://aviation-safety.net/database/record.php?id=19981010-0

${ }^{61}$ https://aviation-safety.net/database/record.php?id=20011004-0
} 


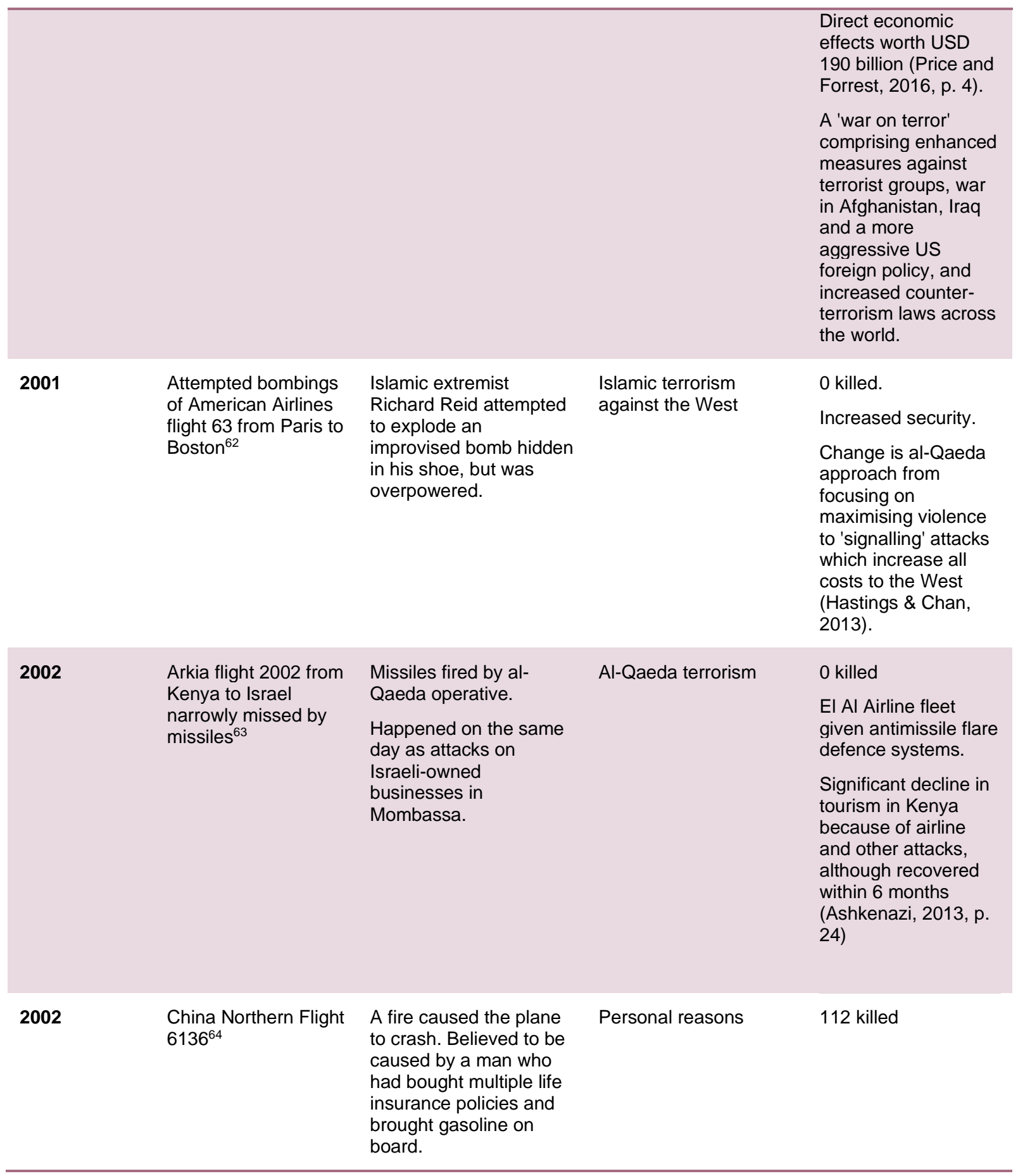

\footnotetext{
62 https://aviation-safety.net/database/record.php?id=20011222-0

${ }^{63} \mathrm{https}: / /$ aviation-safety.net/database/record.php?id=20021128-1

${ }^{64}$ https://aviation-safety.net/database/record.php?id=20020507-1
} 


\begin{tabular}{|c|c|c|c|c|}
\hline 2002 & $\begin{array}{l}\text { New Orleans } \\
\text { shooting } 65\end{array}$ & $\begin{array}{l}\text { A man killed two people } \\
\text { in the airport terminal } \\
\text { before being } \\
\text { overpowered }\end{array}$ & $\begin{array}{l}\text { Unknown personal } \\
\text { motivations. }\end{array}$ & $\begin{array}{l}2 \text { killed } \\
\text { Calls for more armed } \\
\text { guards in airports. }\end{array}$ \\
\hline 2004 & $\begin{array}{l}\text { Siberia Airlines } 1047 \\
\text { and Volga- } \\
\text { Aviaexpress Flight } \\
1303^{66}\end{array}$ & $\begin{array}{l}\text { Explosive devices went } \\
\text { off on the two planes on } \\
\text { the same day. } \\
\text { A ticket agent was found } \\
\text { to have taken a bribe to } \\
\text { allow the bomber to } \\
\text { board within ID. The } \\
\text { bombers were assessed } \\
\text { by a police officer who } \\
\text { found no bombs and } \\
\text { was later imprisoned for } \\
\text { negligence (Price \& } \\
\text { Forrest, 2016, p. 90) }\end{array}$ & $\begin{array}{l}\text { Chechen separatists } \\
\text { claimed responsibility. } \\
\text { Chechen-Russian } \\
\text { conflict. }\end{array}$ & $\begin{array}{l}44 \text { and } 46 \text { killed. } \\
\text { Increased passenger } \\
\text { security at Russian } \\
\text { airports (inc. more } \\
\text { searches and body } \\
\text { imaging devices). }\end{array}$ \\
\hline 2006 & $\begin{array}{l}\text { Failed al-Qaeda } \\
\text { plot }^{67}\end{array}$ & $\begin{array}{l}\text { Intelligence services } \\
\text { foiled a plot to destroy } \\
\text { airliners headed to the } \\
\text { US using liquid } \\
\text { explosives. }\end{array}$ & Al-Qaeda terrorism & $\begin{array}{l}\text { Temporary cessation } \\
\text { of UK air traffic } \\
21 \text { arrested } \\
\text { Prohibition of liquids } \\
\text { in cabin }\end{array}$ \\
\hline 2007 & $\begin{array}{l}\text { Vehicle-borne } \\
\text { explosive driven into } \\
\text { Glasgow Airport }\end{array}$ & $\begin{array}{l}\text { Bollards in front of the } \\
\text { building were effective } \\
\text { (Price \& Forrest, 2016, } \\
\text { p. 89) }\end{array}$ & Terrorism & 1 killed. \\
\hline 2009 & $\begin{array}{l}\text { Northwest Airlines } \\
\text { Flight } 253^{68}\end{array}$ & $\begin{array}{l}\text { A Nigerian national } \\
\text { attempted to set-off an } \\
\text { explosive device. } \\
\text { Nicknamed the } \\
\text { 'underwear bomber' }\end{array}$ & Islamic terrorism & $\begin{array}{l}0 \text { killed. } \\
\text { More security } \\
\text { searches. }\end{array}$ \\
\hline 2010 & $\begin{array}{l}\text { Failed trans Atlantic } \\
\text { plot }^{69}\end{array}$ & $\begin{array}{l}\text { Bombs found in } \\
\text { passenger plane cargo } \\
\text { holds between Yemen } \\
\text { and the US, hidden in } \\
\text { printers. }\end{array}$ & Islamic terrorism & $\begin{array}{l}\text { Toner cartridges } \\
\text { banned from } \\
\text { passenger planes }\end{array}$ \\
\hline
\end{tabular}

\footnotetext{
65 https://www.wlox.com/story/793093/airport-shooting-in-new-orleans-injures-two/

66 https://aviation-safety.net/database/record.php?id=20040824-0

67 https://en.wikipedia.org/wiki/2006_transatlantic_aircraft_plot

68 https://aviation-safety.net/database/record.php?id=20091225-0

69 https://en.wikipedia.org/wiki/2010_transatlantic_aircraft_bomb_plot

70 https://www.france24.com/en/20101101-global-air-security-yemen-bomb-attack-usa-britain-terrorism-cargo
} 


\begin{tabular}{|c|c|c|c|c|}
\hline 2011 & $\begin{array}{l}\text { Bombing in airport } \\
\text { terminals in } \\
\text { Moscow }^{71}\end{array}$ & Suicide bombing & $\begin{array}{l}\text { Islamic extremism or } \\
\text { Caucasian nationalism. }\end{array}$ & 35 killed \\
\hline 2013 & $\begin{array}{l}\text { Mozambique Airlines } \\
\text { flight } 470 \text { crashed by } \\
\text { pilot }\end{array}$ & $\begin{array}{l}\text { Deliberately crashed by } \\
\text { the pilot (Nori, 2020, p. } \\
\text { 79). }\end{array}$ & Personal reasons & 33 killed \\
\hline 2013 & $\begin{array}{l}\text { Shootings at Los } \\
\text { Angeles International } \\
\text { Airport }^{72}\end{array}$ & $\begin{array}{l}\text { The shooter was } \\
\text { wounded by airport } \\
\text { police (Price and } \\
\text { Forrest, 2016, p. 90). }\end{array}$ & & 1 killed \\
\hline \multirow[t]{3}{*}{2014} & \multirow{3}{*}{$\begin{array}{l}\text { Malaysia Airlines } \\
\text { flight MH17 shot } \\
\text { down }\end{array}$} & \multirow{3}{*}{$\begin{array}{l}\text { Shot down with a } \\
\text { Russian-made Buk } \\
\text { missile belonging to a } \\
\text { Russian brigade in } \\
\text { territory controlled by } \\
\text { Russian-backed } \\
\text { Ukrainian separatists } \\
{ }^{74}\end{array}$} & \multirow{3}{*}{$\begin{array}{l}\text { Russian invasion of } \\
\text { Ukraine. }\end{array}$} & 298 killed \\
\hline & & & & $\begin{array}{l}\text { The Netherlands and } \\
\text { Australia have } \\
\text { pointed to Russian } \\
\text { responsibility and a } \\
\text { Dutch court will try } 4 \\
\text { Russian citizens in } \\
\text { absentia. Increased } \\
\text { tensions between } \\
\text { Russia and the West. }\end{array}$ \\
\hline & & & & $\begin{array}{l}\text { Calls for more } \\
\text { caution from airlines } \\
\text { flying over conflict } \\
\text { zones (which are not } \\
\text { always closed to air } \\
\text { traffic by conflict } \\
\text { parties). }\end{array}$ \\
\hline \multirow[t]{2}{*}{2014} & \multirow{2}{*}{$\begin{array}{l}\text { Malaysia Airlines } \\
\text { flight } 370 \\
\text { disappeared }\end{array}$} & \multirow[t]{2}{*}{ Yet to be found } & \multirow{2}{*}{$\begin{array}{l}\text { Second major disaster } \\
\text { for Malaysia Airlines in } \\
\text { a year. }\end{array}$} & 239 missing \\
\hline & & & & $\begin{array}{l}\text { Contributed to } \\
\text { Malaysia Airlines' } \\
\text { struggles and near } \\
\text { bankruptcy, before it } \\
\text { was taken over by a }\end{array}$ \\
\hline
\end{tabular}

\footnotetext{
${ }^{71}$ https://www.theguardian.com/world/2011/jan/24/domodedovo-airport-bombing-moscow

72 https://en.wikipedia.org/wiki/2013_Los_Angeles_International_Airport_shooting

${ }^{73}$ https://www.bbc.co.uk/news/world-europe-28357880

${ }^{74}$ https://www.onderzoeksraad.nl/en/page/3546/crash-mh17-17-july-2014
} 


\begin{tabular}{|c|c|c|c|c|}
\hline & & & & $\begin{array}{l}\text { sovereign wealth } \\
\text { fund. } .^{5}\end{array}$ \\
\hline 2014 & 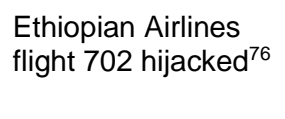 & $\begin{array}{l}\text { Co-pilot hijacked the } \\
\text { plane as part of a } \\
\text { request for asylum }\end{array}$ & $\begin{array}{l}\text { Personal/political } \\
\text { reasons. }\end{array}$ & 0 killed \\
\hline \multirow[t]{3}{*}{2015} & \multirow{3}{*}{$\begin{array}{l}\text { Germanwings flight } \\
9525\end{array}$} & \multirow[t]{3}{*}{ Suicide of pilot } & \multirow{3}{*}{$\begin{array}{l}\text { Mental illness. Self- } \\
\text { reporting of symptoms } \\
\text { and a fear or losing his } \\
\text { licence without } \\
\text { sufficient insurance } \\
\text { means the mental } \\
\text { illness of the pilot was } \\
\text { not known. }{ }^{77}\end{array}$} & 150 killed \\
\hline & & & & $\begin{array}{l}\text { Changes to } \\
\text { Lufthansa policy, and } \\
\text { various countries' } \\
\text { national laws, } \\
\text { stipulating that two } \\
\text { pilots need to be in } \\
\text { the cockpit at any } \\
\text { one time. }\end{array}$ \\
\hline & & & & $\begin{array}{l}\text { Calls for more } \\
\text { psychological testing } \\
\text { of pilots. }{ }^{78}\end{array}$ \\
\hline \multirow[t]{3}{*}{2015} & \multirow{3}{*}{$\begin{array}{l}\text { Metrojet flight } 9268 \\
\text { from Egypt }\end{array}$} & \multirow{3}{*}{$\begin{array}{l}\text { Terrorism - improvised } \\
\text { explosive devise. } \\
\text { Bombers aided by } \\
\text { airport workers (Trends } \\
\text { in Aviation Terrorism, } \\
\text { 2016) }\end{array}$} & \multirow[t]{3}{*}{ Islamic State terrorism } & 224 people killed. \\
\hline & & & & $\begin{array}{l}\text { Cessation of } \\
\text { passenger flights by } \\
\text { many countries }\end{array}$ \\
\hline & & & & $\begin{array}{l}\text { Tourism in Egypt } \\
\text { significantly reduced. }\end{array}$ \\
\hline 2015 & $\begin{array}{l}\text { New Orleans } \\
\text { machete attack }{ }^{81} \\
\text { (Price and Forrest, } \\
2016, \text { p. 50). }\end{array}$ & $\begin{array}{l}\text { A man attacked the } \\
\text { airport with a machete } \\
\text { before being shot. He } \\
\text { also had Molotov }\end{array}$ & Unknown motive & $\begin{array}{l}\text { Attacker shot and } \\
\text { killed. }\end{array}$ \\
\hline
\end{tabular}

\footnotetext{
75 https://www.theguardian.com/business/2015/jun/01/malaysia-airlines-technically-bankrupt-christoph-muellercuts-boss

${ }^{76}$ https://www.bbc.co.uk/news/world-europe-26222674

77 https://aviation-safety.net/database/record.php?id=20150324-0

78 https://ec.europa.eu/transport/sites/transport/files/modes/air/news/doc/2015-07-17-germanwingsreport/germanwings-task-force-final-report.pdf

79 https://aviation-safety.net/database/record.php?id=20151031-0

${ }^{80}$ https://ospreyflightsolutions.exposure.co/45bfc62ef9dad7c5df70ba594625c83a

81 https://www.nola.com/news/crime_police/article_8fb8a5ab-8a3c-5d19-b5ad-2dfaf515676f.html
} 
cocktails.

\begin{tabular}{|c|c|c|c|c|}
\hline \multirow[t]{2}{*}{2016} & $\begin{array}{l}\text { Dallo airlines D3 } \\
159^{82}\end{array}$ & $\begin{array}{l}\text { An explosive device } \\
\text { blew a hole in the } \\
\text { fuselage and a } \\
\text { passenger fell out and } \\
\text { died. }\end{array}$ & $\begin{array}{l}\text { Islamic terrorism; al- } \\
\text { Shabaab conflict with } \\
\text { government of } \\
\text { Somalia. }\end{array}$ & 1 killed. \\
\hline & & $\begin{array}{l}\text { Possibly aided by a } \\
\text { airport worker } \\
\text { (BaMaung et al, 2018) }\end{array}$ & & \\
\hline
\end{tabular}

\begin{tabular}{|c|c|c|c|c|}
\hline 2016 & $\begin{array}{l}\text { Attack at Brussels } \\
\text { Airport }^{83}\end{array}$ & Suicide bombers & $\begin{array}{l}\text { Attacks claimed by } \\
\text { Islamic State terrorist } \\
\text { group }\end{array}$ & 35 deaths \\
\hline 2016 & $\begin{array}{l}\text { Attack at Atatürk } \\
\text { Airport in Istanbul }\end{array}$ & $\begin{array}{l}\text { Shooting and suicide } \\
\text { bombers }\end{array}$ & $\begin{array}{l}\text { Turkey has blamed the } \\
\text { Islamic State terrorist } \\
\text { group, although the } \\
\text { group has not claimed } \\
\text { responsibility. The } \\
\text { attackers were from the } \\
\text { Caucasus }^{84}\end{array}$ & $\begin{array}{l}48 \text { killed } \\
\text { Reduction in tourism } \\
\text { to Turkey }\end{array}$ \\
\hline 2018 & $\begin{array}{l}\text { Possible drone } \\
\text { attacks on UAE } \\
\text { airports }^{85}\end{array}$ & $\begin{array}{l}\text { Yemen's Houthis } \\
\text { claimed responsibility } \\
\text { although the UAE } \\
\text { denies this, as do other }_{\text {sources }}{ }^{86}\end{array}$ & War in Yemen & $\begin{array}{l}\text { Possible delays to } \\
\text { flights. } \\
\text { Houthi propaganda. }\end{array}$ \\
\hline 2018 & $\begin{array}{l}\text { Gatwick airport } \\
\text { closed by a drone } \\
87\end{array}$ & $\begin{array}{l}\text { A drone was spotted } \\
\text { near the airport, } \\
\text { although the reason for } \\
\text { flying it was not }\end{array}$ & $\begin{array}{l}\text { Increased drone } \\
\text { ownership among the } \\
\text { general public. }\end{array}$ & $\begin{array}{l}\text { Airport closure for } \\
\text { several days. } \\
\text { Increased attention to }\end{array}$ \\
\hline
\end{tabular}

\footnotetext{
82 https://aviation-safety.net/database/record.php?id=20160202-0

83 https://www.bbc.co.uk/news/world-europe-39350005

${ }^{84}$ https://www.bbc.co.uk/news/world-europe-36670576

85 https://www.bellingcat.com/news/mena/2018/11/07/investigating-houthi-claims-drone-attacks-uae-airports/

${ }^{86}$ https://www.bellingcat.com/news/mena/2018/11/07/investigating-houthi-claims-drone-attacks-uae-airports/

${ }^{87}$ https://www.theguardian.com/uk-news/2020/dec/01/the-mystery-of-the-gatwick-drone
} 
Gatwick and Heathrow spent £5

million on drone defences.

UK legislation on drone ownership and use $^{88}$

\begin{tabular}{|c|c|c|c|c|}
\hline 2019 & $\begin{array}{l}\text { Hijacking of } \\
\text { Bangladesh Airlines } \\
\text { flight } 147\end{array}$ & $\begin{array}{l}\text { The hijacker used a toy } \\
\text { gun and a fake } \\
\text { explosive device. }\end{array}$ & $\begin{array}{l}\text { Increased security } \\
\text { incidents at Dhaka } \\
\text { airport } 89\end{array}$ & $\begin{array}{l}1 \text { killed (suspect) } \\
\text { Revisions to security }\end{array}$ \\
\hline 2020 & $\begin{array}{l}\text { African Express } \\
\text { Airways plane shot } \\
\text { down in Somalia }^{90}\end{array}$ & $\begin{array}{l}\text { Shot with a ZU-23 anti- } \\
\text { aircraft cannon. Possibly } \\
\text { by Ethiopian AMISOM } \\
\text { troops although full facts } \\
\text { unknown }{ }^{91}\end{array}$ & Conflict in Somalia 92 & 6 killed. \\
\hline 2020 & $\begin{array}{l}\text { Shooting down of } \\
\text { Ukraine International } \\
\text { Airlines flight } \\
\text { PS752 }\end{array}$ & $\begin{array}{l}\text { Shot down by Iranian } \\
\text { missiles by mistake }\end{array}$ & $\begin{array}{l}\text { Heightened tensions } \\
\text { following killing of } \\
\text { Iranian General } \\
\text { Soleimani. The plane } \\
\text { was flying close to a } \\
\text { military centre. }\end{array}$ & 176 people killed \\
\hline
\end{tabular}

Source: compiled by author from multiple sources.

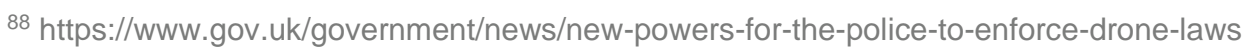

${ }^{89}$ https://ospreyflightsolutions.exposure.co/case-study-the-attempted-hijacking-of-a-biman-airlines-flight-fromdhakas-hazrat-shahjalal-international-airport-24th-february-2019

90 https://aviation-safety.net/database/record.php?id=20200504-0

91 https://aviation-safety.net/database/record.php?id=20200504-0

92 https://www.bbc.co.uk/news/world-africa-52545582

${ }^{93}$ https://www.bbc.co.uk/news/world-middle-east-51073621

94 https://reports.aviation-safety.net/2020/20200108-0_B738_UR-PSR_FACTUAL.pdf
} 


\section{Factors shaping threats}

\section{Types of threat}

This section discusses the different trends in malicious incidents. It discusses both the motivations behind attacks and the means of attacks as threats (or underlying factors shaping threats). It also discusses aviation security measures as shaping the opportunities available to malicious actors.

Underlying many of these threats is the fact that civilian aviation is a high-profile target for terrorists and others. Attacking civilian aviation is attractive to terrorists because: any act is likely to be visible across the globe, can be highly lethal and affect citizens of several countries, is likely to 'depreciate the embodiment of state power that airlines and airports symbolise', make a powerful statement to world leaders, have significant direct and indirect economic consequences, and impede global connectivity. For criminals, civilian aviation provides a means to transport illicit goods or money (Price \& Forrest, 2016). It also provides a high-profile target for those seeking ransoms. For individuals fleeing persecution or poverty, aviation offers the potential of transcending state boundaries (Scott, 2019).

Threats must be seen in the context of the security measures in place to prevent them. Criminologists show how the effect of measures to prevent attacks on potential future attackers varies depending on the aims of the attackers. Some scholars speak of a 'contagion effect' as successful hijackings encourage others, while increased security measures act as a deterrent (Dugan et al., 2005). However, other scholars have found that, in the case of al-Qaeda terrorism, 'hardening' a target (i.e. greater security) does not reduce its attractiveness as terrorists aim to maximise the 'symbolic value' of their attacks, i.e. not just the death toll but the costs of increased vigilance imposed on the target states (Hastings \& Chan, 2013).

Attackers and security planners have adapted over the years (Price \& Forrest, 2016, pp. 60-61). Attacks usually lead to changes in security practices. Over the years, security has increased and measures are usually put in place following a high-profile incident. Complacency, the desire of airport and airline operators to maximise profits, the global nature of air travel, and the evolving tactics of attackers are barriers to effective safety measures. Forrest and Price (2016) show how increased passenger searches in the 1970s did not protect against fake or non-existent bombs. Similarly, the metal detectors and $x$-ray machines widely installed since the 1970 s do not detect semtex, used in the Lockerbie bombing, or liquid explosives. Authorities do not always anticipate changes, or implement changes in a timely manner after failed attacks (Price and Forrest, 2016, p. 91). Increased security on planes has possibly led to more attacks on airports. It is unclear how protected the aviation system is from cyber attacks.

\section{Trends}

Between 1930 and 1979, there were a number of hijackings by individuals escaping persecution. For instance, there were a number of Cubans fleeing Cuba, or leftists fleeing America to Cuba, in the period and beyond (Klenka, 2019, p. 42). By the 1960s, terrorist groups began to use hijackings to bargain and gain attention. The implementation of passenger and baggage screening with the US Anti-Hijacking Act in 1974 led to a decrease of hijackings and bombings, particularly in the US (Price \& Forrest, 2016, p. 46). In the mid-1970s, measures against hijacking became stronger in a number of states, including armed guards on US planes and tactical units within German and Soviet security forces (Scott, 2019, p. 240). Measures to prevent 
hijackings by asylum seekers were also put in place, including an extradition treaty between Cuba and the US in the early 1970s (Price \& Forrest, 2016, p. 48). It is estimated that $41 \%$ of hijackings and $24 \%$ of bombings were foiled in the period 1977-1986, a significant increase on the previous decade, although the success rate fell significantly afterwards (Merari, 1998, p. 21).

Between 1980 and 1990. There were fewer incidents in the US, but US-flagged airlines overseas were attacked. Some of the deadliest attacks occurred, including Pan Am flight 103 and Air India flight 182. Price and Forest $(2016$, p. 47) argue that these two events led the airline industry to focus on the threat of bombings and neglect hijacking until the 2001 attacks on the US. Between 1990 and 2001, attacks on aviation decreased significantly (Price \& Forrest, 2016, p. 61). The 2001 attacks on the US prompted significant increases in aviation security, including armoured cabin doors, although aeroplanes and airports remained important targets for terrorist groups. Other threats, such as suicidal aircrew, or missiles in conflict zones, remained.

Duchesneau and Langlois (2017) compiled a database of 'acts of unlawful interference' on civil aviation from 1931 to 2016 . They categorised attacks as ground attack, hijacking, sabotage and suicide mission. In total, they found 2,071 acts of unlawful interference (635 of which are 'definitively' terrorist) causing 9,508 deaths. They divide the acts into:

- Ground attack 536 (1,865 deaths);

- Hijacking 1,308 (814 deaths);

- Sabotage 174 (2,829 deaths),

- Suicide mission 53 (4,000 deaths)

There have been definite falls in incidents relative to the number of civilian flights over the decades. The risk of civilian air travel has declined in the period surveyed: 'since 1970 the fatal accident rate for U.S. airlines has dropped over $90 \%$ while traffic in the air has soared' (McCrie \& Haas, 2018). In addition, 'statistics point to a decrease in the number of terrorist attacks since 2003, while air traffic has grown at about 5 per cent annually during the same period' (Duchesneau \& Langlois, 2017, p. 351). Nevertheless, a single attack can have a significant effect. Moreover, the levels of risk and safety are uneven, with some areas of the world facing higher risk, as well as emerging threats. An attack can dent a country's tourism income in the short-term.

There is a negative correlation between the method of attack and the number of people killed. Of the four methods surveyed by Duchesne and Langlois (2017) - ground attack, hijacking, sabotage, suicide mission - the most frequently used (hijacking, 1,308 incidents) has killed the fewest people (814); whereas the least common (suicide, 53 incidents), has produced the most deaths (see also Price and Forrest, 2016, p. 49). This may be explained by a number of factors, including that hijackers often intend to use civilians as leverage rather than killing them as an end, and the large number killed in the suicide attacks on the World Trade Centre in September 2001. 


\section{Terrorism}

A significant number of hijackings and bombings since the 1960s can be attributed to what may broadly be termed terrorist groups. ${ }^{95}$ These attacks have a variety of motives and aims. Some of the most prevalent are:

- Calling for the release of political prisoners.

- Using the hostages to demand a ransom or something else from authorities.

- The airing of grievances.

- Attacking passengers or airlines linked to a particular nationality or religion (e.g. Israel or Jews).

- The destruction of sites of symbolic importance (e.g. the World Trade Center, or an aeroplane).

In the 1960s and 1970s, Palestinian groups were prominent, partly because the global, transborder nature of air travel allowed them to effectively highlight the plight of stateless Palestinians through hijacking (Scott, 2019, p. 224). The increasing hardening of attitudes against hijacking saw the Palestinian Liberation Organisation turn away from the method, but other groups such as the Abu Nidal organisation continued to use it. The development of anti-terrorism units within national police, as well as policies such as Israel's of accompanying taxiing aircraft with armed vehicles, reduced the threat of hijackers. Anti-hijacking tactics developed, such as seeking to stay on the ground, or for aircrew to escape so that the plane could not be flown. States became less willing to negotiate. All of these changes meant hijacking was less likely to be successful.

The use of bombs and suicide bombers presents a different threat to hijackers, as the former often do not aim to negotiate. Islamic terrorist organisations have sought to bomb and crash civilian aeroplanes and states have attacked planes such as North Korea's 1987 attack on Korean Air Flight 858, and the 1988 Lockerbie bombing by Libya. Despite increased security and awareness, high profile incidents such as the bombing of Air India 181 or the Lockerbie bomb, and the 9/11 attacks, highlighted flaws and lapses in security procedures. Terrorists have increased attacks on airports, and shown interest in technologies such as drones (Trends in Aviation Terrorism, 2016). 'Lone wolf' terrorists, without the resources of an organised group, may focus on attacking an airport terminal with a gun or knife (Feldman, 2020, p. 52). However, significant attacks on airports also occurred in previous decades such as those on Rome and Vienna airports in 1985.

\section{Individual}

A number of security incidents can be attributed to individuals with what may be termed personal motivations or drivers for deliberately attacking planes. This includes committing suicide by crashing a plane or attacking other members of staff (Nori, 2020; Price \& Forrest, 2016, pp. 82-83).

A second individual factor is airport or airline staff who accept bribes or allow security threats to pass through security (Trends in Aviation Terrorism, 2016). Staff often bypass security checks and are therefore a significant risk (BaMaung et al., 2018). For example, the

\footnotetext{
${ }^{95}$ Some, but not all, of these groups may be designated terrorist groups by particular states.
} 
attacks on Siberian Airlines flights in 2004 were facilitated by the bribing of a member of airport staff.

Although the reasons behind each person's actions are unique, ranging from marital problems to grievances against their employer and mental illness, there are a number of broad factors used to explain such incidents. These drivers include combinations of mental illness, financial motives, pride, etc. The literature points to a number of potential factors shaping this threat:

- Airline screening procedures and psychological support.

- National airline cultures, remuneration and employment rights. This is sometimes pointed to as a reason why a pilot suffering from mental illness does not declare it. If they are likely to lose their licence to fly or financial security through a lack of social safety nets, they are more likely not to declare a problem. The role of honour and shame may also be a contributing factor (Trends in Aviation Terrorism, 2016).

- The working conditions of airline pilots, particularly lack of sleep, are shown to have a possible effect on levels of depression. However, the links are weak (Pasha \& Stokes, 2018).

Examples include the pilot-induced crashes of Japan Airlines flight 350 in 1982 or Egypt Air flight 990 in 1999 likely due to fears about job security; the likely ideologically driven hijacking of (Taiwanese) China Airlines 334 freight plane in 1986 to go to the People's Republic of China; and the Germanwings crash of 2015.

\section{Asylum}

A number of individuals have hijacked planes as a way to seek asylum in another country. This was particularly the case for individuals living in states restricting exit such as the USSR (Scott, 2019). Western publics and sometimes governments pushed for these individuals to be granted asylum, or were at least reluctant to send them back to the USSR. However, by the 1970s, global laws and conventions against hijacking encouraged extradition and stronger measures from individual states determined to prevent hijackings, like those undertaken by Palestinian groups, from disrupting air travel saw a hardening against this type of escape (Scott, 2019).

Examples include attempts by citizens of the Soviet Union or Warsaw Pact countries to seek asylum in the West and Cubans in America in the 1960s and 70s; Americans seeking asylum in Cuba; the hijacking of Aeroflot flight 3729 in 1988 by a family demanding to go to London; and the co-pilot's hijacking of Ethiopian Airlines flight 702 in 2014 in order to make an asylum claim. The hijacking of Xiamen Airlines flight 8301 in 1990 by a man wanting to claim asylum in Taiwan is another example.

\section{Conflict}

A number of security incidents have occurred near conflict zones. In some cases, a civilian aeroplane may be misidentified as a threat and hit with a missile. In others, a plane may be deliberately targeted. A number of civilian airliners were hit by missiles in the wars in Angola, Sudan and Ukraine, for instance. Since 1938, about 80 incidents featuring the shooting down of a commercial airliner have occurred (Klenka, 2019, p. 60). This includes attacks in conflict zones by state actors and non-stated armed groups (including terrorists) and by these actors outside of conflicts. There have been calls for planes to be routed away from conflict zones more 
systematically. Currently the decision is made by states and airlines and is therefore not consistently applied.

The tensions surrounding wars can also increase the likelihood of an unintentional, fatal attack. During the Iran-Iraq war, the US, not a formal combatant, shot down Iran Air Flight 655, killing all of its passengers, after its battleship misidentified the airliner as a threat. Similarly, the recent shooting down of Ukraine International Airlines flight PS752 in 2020 by an Iranian missile shows the dangers created by tensions. The Iranians identified the plane as a threat as it passed near to a military facility, at a time of heightened tensions in Iran following the assassination of an Iranian general. The Soviet air force shot down Korean Air flight 902 in 1978 and Korean Air Lines 007 in 1983, after the airliners strayed into Soviet airspace. While navigation errors and a lack of GPS are a cause, the Cold War tensions also likely contributed. Recently, tensions between India and Pakistan have led to precautionary measures. In 2019, Pakistan closed most of its airspace (February to July) and the US, European, UK, German and French have issued warnings to civilian aeroplanes. ${ }^{96}$

Rebel groups with less capacity to identify threats, less coherent command structures, or less chance of winning through conventional means, may be more likely to attack a civilian aircraft. This may be facilitated by increased supply of surface-to-air missiles during a conflict, for example (Ashkenazi, 2013, p. 16). The Polar 3 scientific plane was hit by a missile fired by Western Saharan separatists in 1985, after it was misidentified as a threat. Georgian civilian planes were hit by a missile fired by Abkhazian separatists in 1993. A recent report points to the possibility of rockets fired by the Tigray People's Liberation Front (TPLF) in Ethiopia hitting civilian aircraft or airports. ${ }^{97}$ This threat is stronger in fragile or conflict-affected contexts and 'manpad' surface-to-air missiles have never been used in developed countries.

In some cases, civilian airliners are targeted deliberately as in Air Rhodesia flight 825 in 1978 and 827 in 1979, which were shot down by the Zimbabwe People's Revolutionary Army during conflict between colonial and anti-colonial forces, partly to target senior opposition figures. It is unclear how deliberate the shooting down of Malaysian Airlines flight $\mathrm{MH} 17$ by a missile belonging to a Russian brigade in Ukraine was, although the ongoing war and the Russian use of semi-independent fighting units may be contributing factors.

Civilian aircraft have been destroyed on the ground, usually without fatalities, during conflicts such as the Lebanese war, the Gulf War, the NATO war in Libya.

\section{Organised crime}

\section{Organised crime can also create and expose weaknesses in aviation security that} terrorists might exploit. According to one report, 'international drug trafficking through airports, which is often facilitated by corruption, is the structural basis on which terrorists might also consummate their deadly attacks.' It points to 'indifference' from South American aviation authorities, which allows the movement of illicit goods, and potential threats to aircraft (Drug Trafficking and Terrorism, 2017).

\footnotetext{
${ }^{96}$ https://ospreyflightsolutions.exposure.co/indiapakistan-navigating-airspace-disruption-amid-heightenedtensions
}

${ }^{97} \mathrm{https}: / /$ ospreyflightsolutions.exposure.co/analysis-tigray-conflict 
Several attacks on civilian planes can be directly linked to the illegal drug trade in South America. For instance, in 1989, the Medellin cartel destroyed Avianca Flight 203 in Colombia, possibly with the motive of killing an informant on the plane.

\section{Cyber}

Cyber attacks pose a significant threat to civilian aviation. A recent article notes that a number of computer systems used in aviation are hackable: reservation systems, flight traffic management systems, access control management systems, departure control systems, passport control systems, cloud-based airline data storage, hazardous materials transportation management, cargo handling and shipping (Four Threats to Aviation Security - and Four Responses, 2016; Ken-Dror \& Gross, 2019). It also notes that aeroplanes will usually use flight control systems, GPS-based navigation systems, fuel gauges and fuel consumption systems, and maintenance computers, among others, that are hackable (Four Threats to Aviation Security - and Four Responses, 2016; Ken-Dror \& Gross, 2019).

The threat is considerable because many people have the means to hack these systems (Four Threats to Aviation Security - and Four Responses, 2016). A hacker can remotely attack plane navigation systems and have taken control of planes' movements using relatively simple technology like laptops and smartphones (Ken-Dror \& Gross, 2019, pp. 139-140).

A cyber attack may also be harder to detect if, for example, terrorists divert a plane without the pilots noticing. Malicious computer code can also be put onto an aeroplane or systems long before it is activated (Ken-Dror \& Gross, 2019, p. 166)

The systems involved in civilian aviation, such as check-in or air traffic control, are also vulnerable. Airport systems may not be run by each airport, e.g. check-in systems operated multinationally, meaning that an attack on a big operator may affect several airports (Ken-Dror \& Gross, 2019). There are several examples of cyber attacks affecting air operations, including:

- A 2006 cyber attack saw the FAA shut down air traffic control systems in Florida (KenDror \& Gross, 2019, p. 135) .

- In 2013, a cyber attack against Istanbul airport affected the passport control system and delayed flights (Ken-Dror \& Gross, 2019, p.136).

- In 2017, Ukraine's Boryspil International Airport saw its computers and departure boards disabled by a cyber attack (Ken-Dror \& Gross, 2019, p.136).

- 'On August 20, 2008, Spanair Flight 5022 (JK5022) from Barcelona's El Prat airport (via Madrid's Barajas airport) to Gran Canaria airport in Spain, crashed minutes after taking off from Madrid airport. One hundred and fifty-three people died, and 18 survived. Two years later, in a hardly circulated notice, Spanair reported that the company's main computer, which recorded aircraft malfunctions, had been contaminated with malicious computer programs and, therefore, might not have recognized the airplane's problems before take-off' (Ken-Dror \& Gross, 2019, p. 137).

- 'In June 2015, the Polish airline LOT reported a cyber-attack that affected its ground operation systems, which prevented the LOT personnel from developing flight plans' (Ken-Dror \& Gross, 2019, p. 138). 


\section{UAVs}

Unmanned aerial vehicles are seen as a growing threat to civilian aviation. For terrorists, drones are 'lower risk and less costly than sending operatives aboard a commercial plane to disrupt its flight' (Chávez, 2020). UAVs are used widely by civilians including hobbyists, so not all incidents are straightforwardly 'malicious'.

UAVs have been responsible for a number of incidents in the US and UK, although it is not always clear what the intention of the UAV users was. Using internet searches and NASA and FAA databases, Pyrgies (2019) has identified 139 UAV incidents within 35 kilometres of civilian airports between May 2014 and May 2018. Incidents covered are: near mid-air collisions (NMAC), mid-air collisions (MAC), airspace closures, jetlining sightings and airport indoor sightings. It includes 13 incidents in UK airports, and 24 at US airports. Dubai airport had to close three times, London Gatwick twice, and Sharjah International, Chengdu Shuangliu International, Chongqing International and Auckalnd International all had to close once in the period. He notes that, 'in 137 cases out of 139 , neither the pilot nor the UAV could be identified by the forensic investigation that has been systematically launched after the incident.' In the UK in 2018, there were around 15 'airprox' incidents - defined as incidents where drones come close enough to planes to possibly compromise safety - per month (Chavez, 2020, p. 37). There have been a number of near misses. For example, in 2014 an American Airlines Group regional jet in Florida nearly collided with a drone at 2,300 feet (Maddox \& Stuckenberg, 2015).

Terrorists have planned to use drones against civilian aviation. Yemeni Houthi groups have reported attacking civilian airports in the UAE, although the UAE denies this.

\section{References}

Ashkenazi, M. (2013). MANPADS A Terrorist Threat to Civilian Aviation? https://www.bicc.de/uploads/tx_bicctools/BICC_brief_02.pdf

David BaMaung, David Mcllhatton, Murdo MacDonald \& Rona Beattie (2018) The Enemy Within? The Connection between Insider Threat and Terrorism, Studies in Conflict \& Terrorism, 41:2, 133-150, DOI: 10.1080/1057610X.2016.1249776

Chávez, K. (2020). Off the Shelf: The Violent Nonstate Actor Drone Threat. https://www.defensehere.com/eng/defense-industry/off-the-shelf--the-violent-nonstate-actordrone-threat/76519

Drug Trafficking and Terrorism: Identical structures for criminal opportunities utilising aviation I Transport Security International Magazine. (2017). https://tsi-mag.com/drug-trafficking-terrorismidentical-structures-criminal-opportunities-utilising-aviation/

Duchesneau, J., \& Langlois, M. (2017). Airport attacks: The critical role airports can play in combatting terrorism. Journal of Airport Management, 13. https://trid.trb.org/view/1485622

Dugan, L., Lafree, G., \& Piquero, A. R. (2005). Testing a rational choice model of airline hijackings. Criminology, 43(4), 1031-1065. https://doi.org/10.1111/j.1745-9125.2005.00032.x

Feldman, D. (2020). Mitigating Potential Lone Wolf Terrorist Attacks Against Aviation Sector Infrastructure [Johns Hopkins]. 
https://jscholarship.library.jhu.edu/bitstream/handle/1774.2/62331/FELDMAN-THESIS-

2019.pdf?sequence $=1$ \&isAllowed $=y$

Four threats to aviation security - and four responses. (2016). World Economic Forum.

https://www.weforum.org/agenda/2016/07/four-threats-to-aviation-security-and-four-responses/

Hastings, J. V., \& Chan, R. J. (2013). Target Hardening and Terrorist Signaling: The Case of Aviation Security. Terrorism and Political Violence, 25(5), 777-797.

https://doi.org/10.1080/09546553.2012.699906

Ken-Dror, D., \& Gross, E. (2019). Cyber Terrorism and Civil Aviation: Threats, Standards and Regulations. Journal of Transnational Law \& Policy, 29.

https://law.fsu.edu/sites/g/files/upcbnu1581/files/JTLP/2019-

2020/\%2804\%29\%20JTLPv29_Feldman-Gross-Article_102820.pdf

Klenka, M. (2019). Major incidents that shaped aviation security. Journal of Transportation

Security, 12, 39-56. https://ideas.repec.org/a/spr/jtrsec/v12y2019i1d10.1007_s12198-01900201-2.html

Maddox, S., \& Stuckenberg, D. (2015, February 24). Drones in the U.S. National Airspace System: A Safety and Security Assessment. Harvard National Security Journal.

https://harvardnsj.org/2015/02/drones-in-the-u-s-national-airspace-system-a-safety-and-securityassessment/

McCrie, R., \& Haas, D. (2018). Why Airline Passenger Screening Will Be With Us Forever: Past, Present, and Prospects for Air Travel Safety. Journal of Applied Security Research, 13(2), 149159. https://doi.org/10.1080/19361610.2018.1422359

Merari, A. (1998). Attacks on civil aviation: Trends and lessons. Terrorism and Political Violence, 19. https://doi.org/10.1080/09546559808427466

Nori, C. H. A. (2020). A review of hijack events by airline employees; with a particular reference to Pilot Suicide Using an Aircraft (PSUA)—An analysis of the triggering factors and current mitigation [London Metropolitan University]. http://repository.londonmet.ac.uk/6132/1/NoriHani_Thesis_v5.5.pdf

Pace, S., Frost, G., Lachow, I., Frelinger, D., Fossum, D., Wassem, D., \& Pinto, M. (1995). The Global Positioning System: Assessing National Policies. RAND Corporation.

https://doi.org/10.7249/MR614

Pasha, T., \& Stokes, P. R. A. (2018). Reflecting on the Germanwings Disaster: A Systematic Review of Depression and Suicide in Commercial Airline Pilots. Frontiers in Psychiatry, 9. https://doi.org/10.3389/fpsyt.2018.00086

Price, J. C., \& Forrest, J. S. (2016). Practical aviation security: Predicting and preventing future threats. Elsevier. https://doi.org/10.1016/B978-0-12-804293-9.00001-1

Scott, E. R. (2019). The Hijacking of Aeroflot Flight 244: States and Statelessness in the Late Cold War*. Past \& Present, 243(1), 213-245. https://doi.org/10.1093/pastj/gty044

Trends in Aviation Terrorism. (2016). International Institute for Counter-Terrorism. https://www.ict.org.il/Article/1757/trends-in-aviation-terrorism\#gsc.tab=0 


\section{Acknowledgements}

We thank the following experts who voluntarily provided suggestions for relevant literature or other advice to the author to support the preparation of this report. The content of the report does not necessarily reflect the opinions of any of the experts consulted.

- Kerry Chávez, Texas Tech University

\section{Key websites}

- Aviation Safety Network: https://aviation-safety.net

\section{Suggested citation}

Kelly, L. (2021). Threats to civilian aviation since 1975. K4D Helpdesk Report. Brighton, UK: Institute of Development Studies. DOI: 10.19088/K4D.2021.019

\section{About this report}

This report is based on six days of desk-based research. The K4D research helpdesk provides rapid syntheses of a selection of recent relevant literature and international expert thinking in response to specific questions relating to international development. For any enquiries, contact helpdesk@k4d.info.

K4D services are provided by a consortium of leading organisations working in international development, led by the Institute of Development Studies (IDS), with Education Development Trust, Itad, University of Leeds Nuffield Centre for International Health and Development, Liverpool School of Tropical Medicine (LSTM), University of Birmingham International Development Department (IDD) and the University of Manchester Humanitarian and Conflict Response Institute (HCRI).

This report was prepared for the UK Government's Foreign, Commonwealth and Development Office (FCDO) and its partners in support of pro-poor programmes. Except where otherwise stated, it is licensed for non-commercial purposes under the terms of the Open Government Licence v3.0. K4D cannot be held responsible for errors, omissions or any consequences arising from the use of information contained in this report. Any views and opinions expressed do not necessarily reflect those of FCDO, K4D or any other contributing organisation.

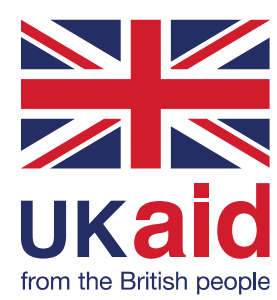

(C) Crown copyright 2021. 


\section{Appendix: Trends in airline fatalities and hijackings, 1975- 2017}

Fatalities from commercial airlines per million passengers, 1975-2017

Aviation fatalities from commercial airlines per million passengers, World, 1975 to 2017

Global number of fatalities from aviation accidents per million air passengers carried. Fatality figures are based on the number on commercial flights (with capacity for 14+ passengers) and do not include corporate jet or military transport incidents.

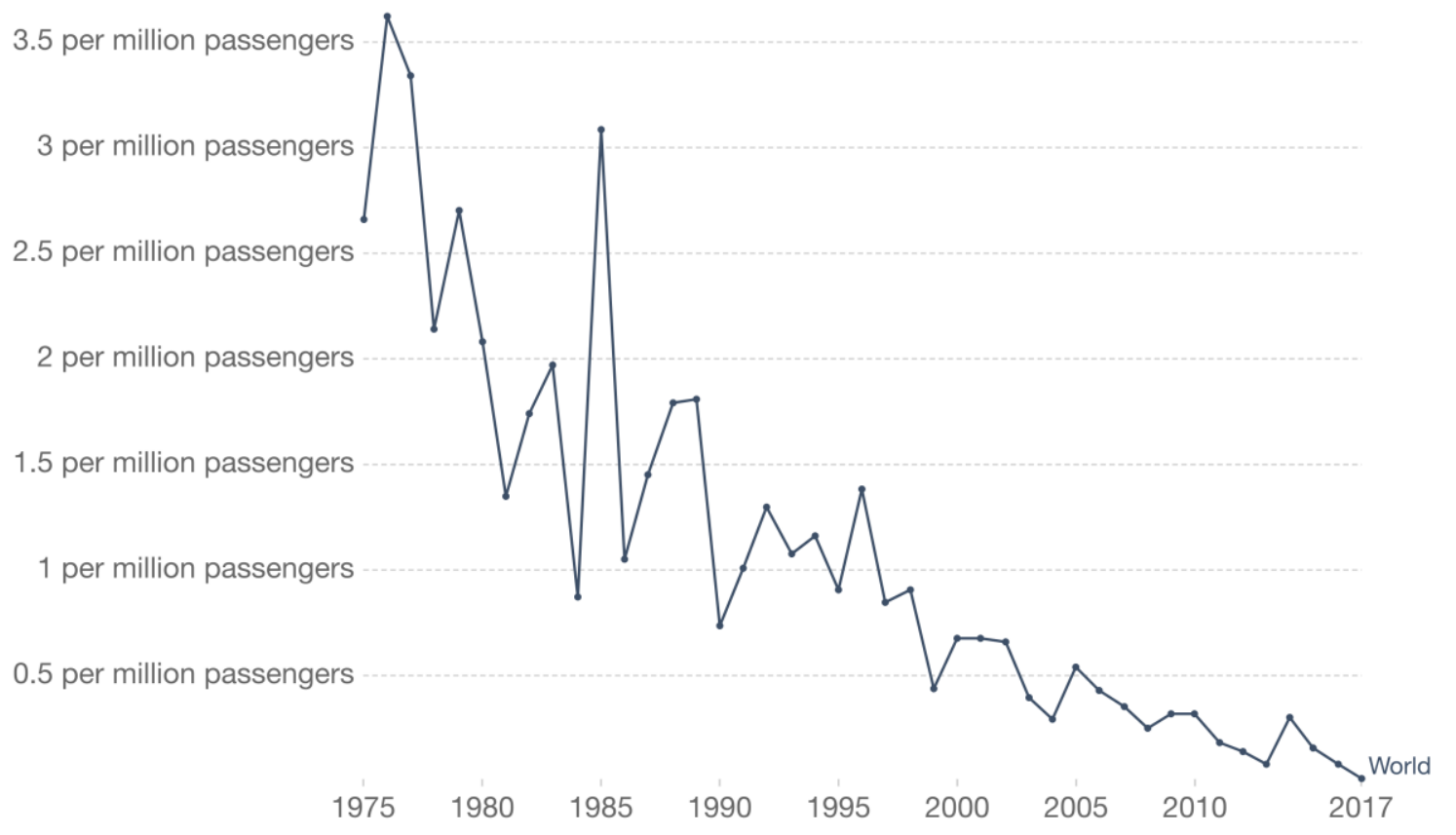

Source: Aviation Safety Network (ASN)

Source: Our World in Data. Licensed under CC BY 4.0. 
Fatalities from aviation accidents and hijackings, global, 1975-201798

Global fatalities from aviation accidents and hijackings

Data is based on cargo and passenger commercial airliners (equipped with capacity for 14+ passengers) and does not include corporate jet and military transport incidents. The fatalities from hijackings are also included in total commercial airliner figures.

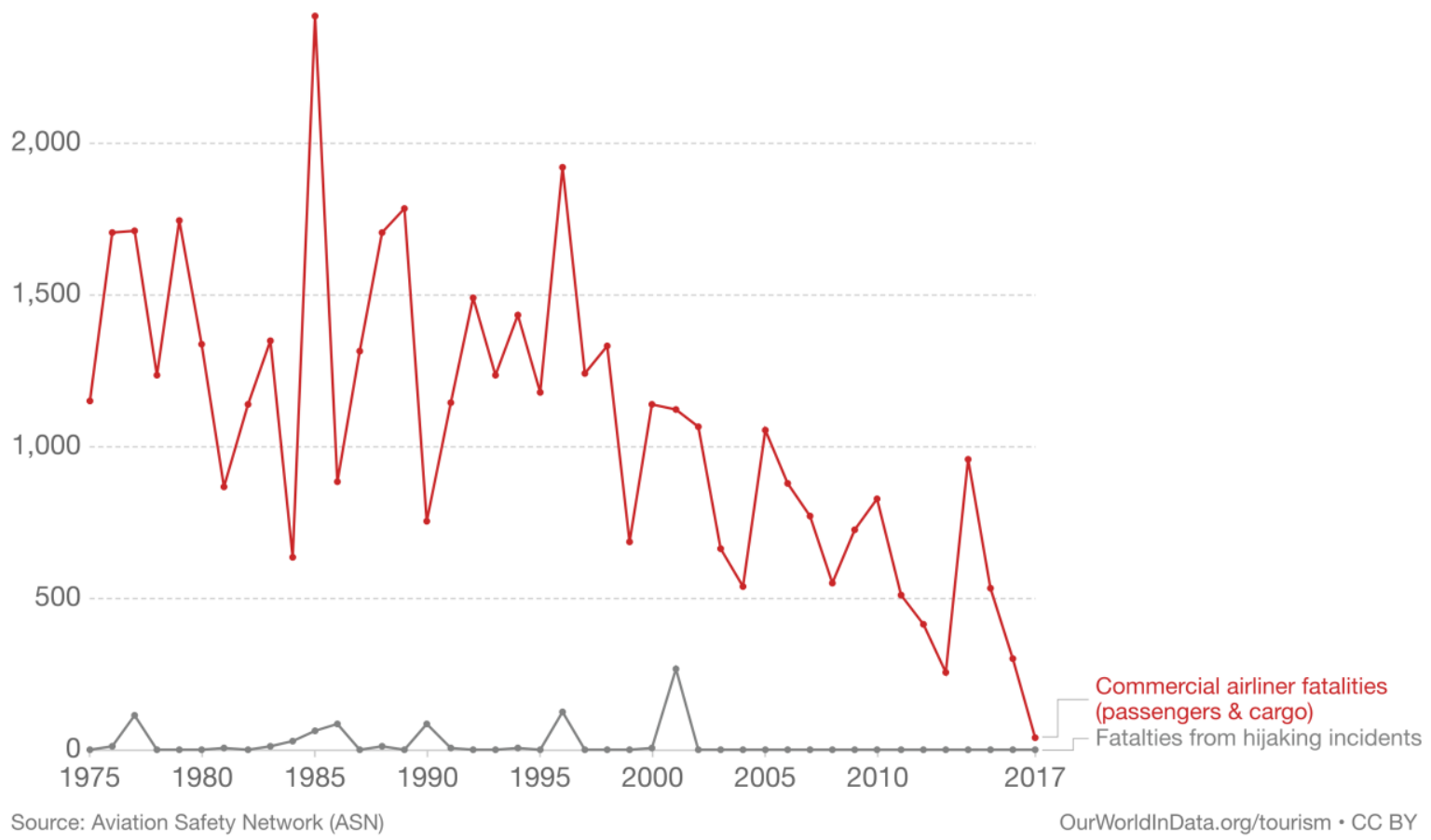

Source: Our World in Data. Licensed under CC BY 4.0.

98 This graph includes fatalities from non-malicious accidents. 
Number of airliner hijackings and fatalities, global, 1975-201799

Global number of airliner hijackings and fatalities

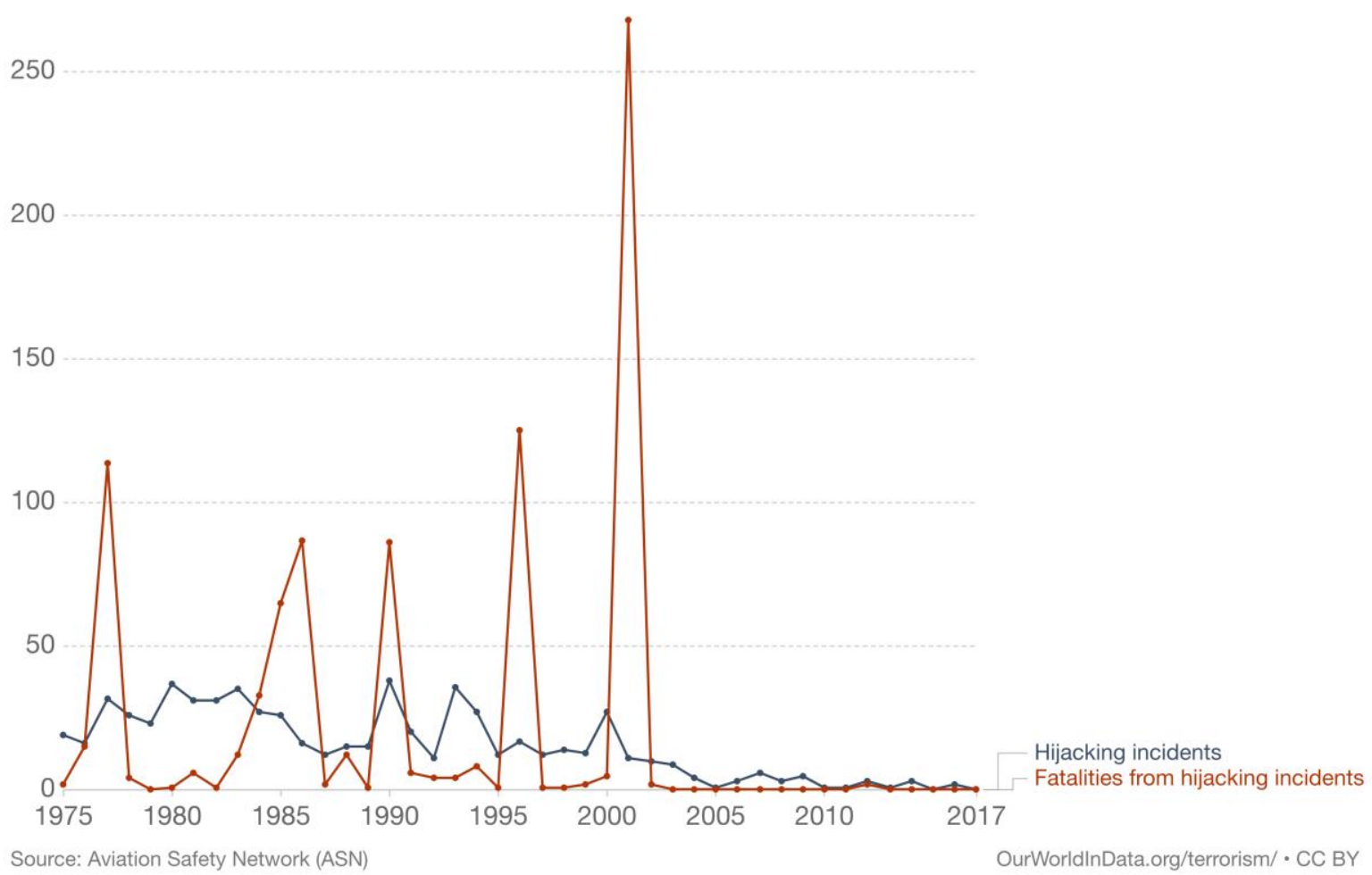

Source: Our World in Data. Licensed under CC BY 4.0.

99 These figures may not tally with those in the table in figure 1, as the table includes bystanders killed by aircraft accidents (e.g. those killed in the World Trade Centre), whereas the graph appears only to include passengers. 